\title{
ANALYSE DES STATISTIQUES DE PÊCHE AUX ENGINS DANS LE BASSIN DU RHÔNE.
}

\author{
SECONDE PARTIE : ÉTUDE DES CAPTURES.
}

\author{
T. CHANGEUX (1) et M. ZYLBERBLAT (2)
}

(1) Laboratoire d'Ecologie des Systèmes Fluviaux, URA CNRS 1451, 1 rue Parmentier, 13200 ARLES, France.

(2) Direction Régionale de l'Environnement Rhône-Alpes, 12 quai Maréchal Joffre, 69002 LYON, France.

Reçu le 21 avril 1993

Accepté le 31 décembre 1993

Received 21 April, 1993

Accepted 31 December, 1993

\section{RÉSUMÉ}

En confrontant les captures déclarées et les autorisations délivrées sur le Rhône et la Saône en 1988 et 1989, on estime pour ces deux années les prélèvements de la pêche aux engins à 238 tan et 273 tan. Les captures sont essentiellement constituées de grands cyprinidés (Abramis brama, Blicca bjoerkna, Rutilus rutilus et Chondrostoma nasus) et de poissons amphihalins comme les muges (Liza ramada en majorité) ou l'anguille (Anguilla anguilla). La pêche professionnelle fluviale représente en moyenne $72 \%$ des captures. Pour certaines espèces comme les aloses (Alosa fallax en majorité), le brochet (Esox lucius), le sandre (Stizostedion lucioperca) et le poisson-chat (Ictalurus melas), les pêcheurs amateurs aux engins et filets prélèvent des quantités comparables à celles des professionnels. De plus, les amateurs sont majoritaires dans les productions de la Saône amont. La biomasse totale des captures du Rhône deltaïque arrive en seconde position derrière celle de la Saône aval, située au centre du bassin. Ce secteur apparaît comme une zone de première importance pour la pratique de la pêche aux engins strictement fluviale en France. Les captures déclarées suivent un rythme saisonnier en relation avec le cycle de l'effort de pêche mis en évidence dans la première partie de l'article (CHANGEUX et ZYLBERBLAT, ce numéro). Les aloses sont capturées pendant la fermeture des autres pêches, au mois de mai. Les muges sont capturés pendant la saison "froide" et la friture de petits cyprinidés (Alburnus alburnus, en majorité) pendant la "saison chaude". Dans la Saône aval, l'analyse des Captures par Unité d'Effort (CPUE) valide la distinction des métiers définis dans la première partie de l'article. L'utilisation des CPUE est envisagée pour suivre les stocks des principales captures du secteur.

Mots-clés : pêcherie, engins, captures, enquête, Capture par Unité d'Effort, bassin du Rhône.

\section{ANALYSIS OF FISHING GEAR FISHERY STATISTICS IN THE RHÔNE RIVER BASIN.}

\section{PART TWO : STUDY OF THE CATCH.}

\section{ABSTRACT}

From the data on fish captures in the Rhône and Saône rivers declared to the fishing authorities in 1988 and 1989, we assessed a harvest from fishing gear of 238 and 272 tons per year, respectively. The captures essentially consist of large cyprinids (Abramis brama, Blicca bjoerkna, Rutilus rutilus and Chondrostoma nasus), euryhaline fishes such as mullets (essentially Liza ramada) and eels (Anguilla anguilla). Commercial (professional) fishing 
represents on average $72 \%$ of the captures. For certain species such as shad (essentially Alosa fallax), pike(Esox lucius), pikeperch (Stizostedion lucioperca) and catfish (Ictalurus melas), recreational fishermen (amateurs) catch quantities similar to the professionals. Moreover, they account for the majority of the yield of the upper Saône. The biomass of the captures in the deltaic Rhône is ranked second behind that of the lower Saône, situated in the center of the basin. This sector is a region of national importance for a strictly fluvial fishery. The declared captures follow a seasonal cycle related to fishing effort as explained in the first part of the paper (CHANGEUX et ZYLBERBLAT, this issue). The shads are captured when the other fisheries are closed in the month of May. The mullets are captured during the "cold" season and the small cyprinids (essentially Alburnus alburnus) are caught during the "warm" season. In the lower Saone, the analysis of the Catch Per Unit Effort (CPUE) shows significant variations between the categories of fishing as described in the first part of the paper. The use of CPUE is suggested in monitoring the stocks of the most important catches of the sector.

Key-words : fishery, fishing gear, catch, survey, Catch Per Unit Effort, Rhône River basin.

\section{INTRODUCTION}

La pêche aux engins dans le bassin du Rhône fait encore partie des usages traditionnels du Domaine Public Fluvial (DPF). Elle comprend deux activités fondamentalement différentes. Une activité commerciale d'exploitation (la pêche professionnelle) et une activité légalement admise uniquement à titre récréatif (la pêche amateur). Cette étude évalue l'utilisation des déclarations effectuées par ces deux catégories de pêcheurs sous la forme de carnets de pêche pour un suivi à long terme des ressources halieutiques du Rhône et de son affluent principal, la Saône. Elle prend pour référence les années d'exploitation 1988 et 1989.

Dans une première partie, nous avons précisé le contexte général de notre étude et exposé les bases méthodologiques de notre approche en nous intéressant plus particulièrement à l'effort de pêche (CHANGEUX et ZYLBERBLAT, ce numéro). Le DPF du bassin rhodanien exploité par la pêche aux engins, présenté en détail dans la première partie, se subdivise en cinq secteurs d'exploitation plus ou moins fréquentés par les pêcheurs (Fig. 4). La portion de la Saône située entre la confluence avec le Doubs et la confluence avec le Rhône (Saône aval) est le secteur le plus exploité du bassin. La Saône amont, en amont de la confluence avec le Doubs, et le Rhône aval, entre la confluence avec la Saône et le Rhône deltaïque, sont les moins fréquentés. Le Rhône amont, en amont de la confluence avec la Saône, et le Rhône deltaïque présentent une fréquentation intermédiaire. D'après CASTELNAUD et BABIN (1990), 34 pêcheurs professionnels ont exercé sur le Rhône et la Saône pendant les deux années qui nous intéressent. Nous estimons le nombre de pêcheurs amateurs à environ 700 .

De 1988 à 1989, 752 carnets ont été retournés, ce qui porte le taux de réponses à $46,8 \%$, catégories de pêcheurs, années et secteurs confondus. Les données étudiées, saisies sur une base mensuelle, totalisent en moyenne 5730 journées de pêche par an. L'étude de l'effort de pêche nous a conduits à distinguer différents métiers : 3 métiers de pêche professionnelle, et 5 métiers de pêche amateur (CHANGEUX et ZYLBERBLAT, ce numéro). Nous avons également proposé de considérer chaque secteur séparément. Ainsi définie, l'utilisation des métiers pour le calcul de Captures par Unité d'Effort (CPUE) reste à évaluer.

Dans cette seconde partie, nous avons ajouté aux données déjà présentées dans la première partie, les captures déclarées par les pêcheurs. Ces captures sont mieux documentées que les données d'effort de pêche, en particulier sur le Rhône (SORNAY, 1932 ; RAMEYE et al., 1976 ; BRAVARD, 1987 ; BOUCHERON, 1980 ; PATTEE, 1988). Les estimations les plus récentes concernent uniquement les pêcheurs professionnels avec une production évaluée à 127 t pour 1989 (BABIN, 1992a). En revanche, aucun calcul de CPUE n'a été tenté sur notre domaine d'étude.

L'objectif de notre travail est, d'une part, d'effectuer une estimation des prélèvements réalisés par les pêcheurs amateurs et professionnels avec une précision acceptable, d'autre 
part, de tenter de déterminer des CPUE permettant de suivre l'évolution des ressources d'une année sur l'autre.

\section{MÉTHODES}

\subsection{Les déclarations de captures}

Les captures sont généralement connues en biomasse par mois de pêche. Elles ont été réparties en sept catégories à partir des déclarations brutes qui n'indiquent pas systématiquement l'espèce (Tableau I). La définition de ces catégories fait intervenir des critères d'exploitation (e.g. espèces cibles bien identifiées, espèces à forte valeur marchande). Elle fait également intervenir la biologie des espèces (e.g. même tolérance visà-vis de la salinité de l'eau, même groupe trophique, préférence écologique commune).

\section{Tableau I : Regroupement des captures en catégories à partir des déclarations brutes.}

Table I : Grouping of the captures by category based on original declarations.

\section{CATÉGORIES DES CAPTURES}

\begin{tabular}{lc}
\hline 1 - Anguille (Anguilla anguilla) & 4 - Petits cyprinidés \\
2 - Autres amphihalins & Friture sans précision \\
Alose feinte (Alosa fallax) en majorité & Ablette (Alburnus alburnus) \\
Muge sp. (Liza ramada) en majorité & Spirlin (Alburnoides bipunctatus) \\
Lamproie marine (Petromyzon marinus) en majorité & Goujon (Gobio gobio) \\
Loup ou Bar commun (Dicentrarchus labrax) & Bouvière (Rhodeus amarus) \\
3 - Grands cyprinidés & 5 - Carnassiers \\
Brème sp. (Abramis brama et Blicca bjoerkna) & Black-bass (Micropterus salmoides) \\
Gardon (Rutilus rutilus) & Brochet (Esox lucius) \\
Hotu (Chondrostoma nasus) & Perche (Perca fluviatilis) en majorité \\
Barbeau fluviatile (Barbus barbus) & Sandre (Stizostedion lucioperca) \\
Carpe toutes variétés confondues (Cyprinus carpio) & - Salmonidés \\
Chevaine (Leuciscus cephalus) & Ombre (Thymallus thymallus) \\
Carpe commune (Cyprinus carpio) & Salmonidés sans autre précision \\
Cyprinidés sans précision & Truite fario (Salmo trutta) en majorité \\
Tanche (Tinca tinca) & - Autres captures \\
Rotengle (Scardinus erythrophthalmus) & Ecrevisse américaine (Orconectes limosus) \\
Vandoise (Leuciscus leuciscus) & Grémille (Gymnocephalus cernua) \\
& Lote (Lota lota) \\
& Perche-soleil ou Canada (Lepomis gibbosus) \\
& Poisson-chat (Ictalurus melas) \\
& Silure glane (Silurus glanis) \\
\hline
\end{tabular}

Une première catégorie concerne l'anguille, qui fait l'objet d'une exploitation professionnelle particulière (BABIN,1992b). Bien que l'anguille d'avalaison soit également capturée de septembre à fin décembre dans le Rhône deltaïque, la distinction n'est pas faite dans les déclarations. Une deuxième catégorie est consacrée aux autres poissons amphihalins ou euryhalins, dont la présence est liée à la proximité de la mer. Ce sont des migrateurs réguliers (alose feinte, lamproie marine) ou des poissons présents toute l'année dans le fleuve (muge, loup). La troisième catégorie rassemble les grands cyprinidés. C'està-dire les poissons susceptibles d'être déclarés sous le terme "blancs". La quatrième catégorie est formée des poissons de petite taille pêchés pour la "friture". Dans ce cas, les espèces concernées sont pour la plupart des cyprinidés (ablette), c'est pourquoi nous lui avons donné l'appellation petits cyprinidés. La cinquième catégorie concerne les poissons prédateurs, traditionnellement appelés carnassiers par les pêcheurs, qui ont une forte valeur commerciale (BABIN,1992b). Du point de vue biologique et commercial, cette 
catégorie se rapproche des salmonidés qui sont également des prédateurs à forte valeur marchande. Cette sixième catégorie a été distinguée dans la mesure où il s'agit surtout d'espèces indicatrices dans la zonation longitudinale des cours d'eau. Enfin, la septième et dernière catégorie regroupe les espèces qui ne font pas l'objet d'une exploitation particulière facilement identifiable, sans avoir de caractéristique écologique fondamentale (écrevisse américaine, poisson-chat, lote, silure). Les déclarations de captures sans précision d'espèce relèvent également de cette catégorie.

\subsection{Analyse des déclarations}

Les déclarations brutes ont été utilisées pour effectuer une étude symétrique de l'étude qualitative spatio-temporelle de l'effort de pêche (CHANGEUX et ZYLBERBLAT, ce numéro). Elle a pour objectif principal de valider notre jeu de données dans son ensemble.

Les répartitions spatiale et temporelle des captures déclarées ont été abordées par deux tableaux établis années et catégories de pêcheurs confondues, de manière à s'affranchir des variations inter-annuelles et des distinctions entre pêcheurs professionnels et amateurs effectuées par ailleurs. La somme des biomasses déclarées par mois a fourni le calendrier des captures. La somme des biomasses déclarées par secteur d'exploitation a permis d'établir leur répartition spatiale. Ces chiffres ont tous été ramenés à 100 carnets, de manière à s'affranchir des variations de fréquentation mensuelles et sectorielles mises en évidence dans la première partie de l'article. Ainsi définis, ce sont deux tableaux de contingence dans lesquels les captures déclarées sont réparties respectivement par mois et par secteur.

La répartition temporelle des captures a été directement comparée avec les saisons de pêches "froide", "chaude" et "intermédiaires" mises en évidence par l'étude de l'effort de pêche (CHANGEUX et ZYLBERBLAT, ce numéro).

La répartition spatiale des captures a été traitée par l'Analyse Factorielle des Correspondances (AFC) du logiciel ADE qui est la méthode adaptée pour les tableaux de contingence (CHESSEL et DOLEDEC, 1991). Les lignes (captures) ayant une contribution absolue supérieure à $10 \%$ de l'inertie du plan ont été retenues comme structurantes. Pour obtenir des groupes de captures, nous avons effectué, en complément, une classification ascendante hiérarchique sur la distance du Chi 2 comme le propose ROUX (1985), en utilisant l'algorithme du saut minimum proposé par le logiciel MacDendro (THIOULOUSE, 1991).

\subsection{Estimation des captures}

Les données de captures manquantes, nécessitant une estimation, se ramènent à deux cas : d'individus :

- les captures n'ont pas été indiquées en poids, mais uniquement en nombre

- le carnet n'a pas été retourné.

Dans le premier cas, le poids des captures a été estimé à partir du poids moyen calculé sur un extrait de captures d'effectifs et de poids connus sur l'ensemble du bassin. Nous avons vérifié que les poids moyens correspondaient bien à des tailles de poissons habituellement capturés avec les engins considérés.

Dans le second cas, le nombre d'autorisations délivrées a permis d'établir une estimation des captures de chaque catégorie de pêcheurs en utilisant les équations relatives à l'échantillonnage stratifié par secteur d'exploitation (CHANGEUX et ZYLBERBLAT, ce numéro). Au niveau du secteur d'exploitation, la même estimation a été obtenue par application des équations de l'échantillonnage aléatoire simple (SCHERRER, 1983).

L'intervalle de confiance de ces résultats, toujours calculé au risque alpha de $5 \%$, se déduit de la variance de l'estimateur. II est borné à gauche par la valeur déclarée lorsque le surplus estimé est inférieur à la taille de l'intervalle. Les intervalles de confiance des moyennes ou des sommes sont obtenus par la somme ou la moyenne des bornes. 


\subsection{Calcul des Captures Par Unité d'Effort}

En remplacement de la notion de population, qui est liée à des considérations biologiques, nous avons retenu celle du stock halieutique associée à l'exploitation telle que la définissent LAUREC et LE GUEN (1981). II s'agit d'une unité de gestion correspondant à la partie exploitable d'une unité biologique (stade de développement particulier, espèce, groupe d'espèces...) présentant une dynamique et une exploitation homogène. Pour constituer un indice annuel d'abondance des stocks halieutiques, nous avons ramené les captures déclarées à un effort de pêche standard, ce qui revient à calculer des Captures Par Unité d'Effort (CPUE).

Pour illustrer les possibilités offertes par une application de cette approche à la pêcherie multi-spécifique et multi-engins du bassin rhodanien, nous avons choisi d'étudier plus particulièrement la Saône aval qui est le secteur le plus intensément exploité du bassin. En l'absence d'informations précises sur les espèces cibles, les stocks retenus ont été déduits de l'analyse de la répartition inter-sectorielle des captures et d'une étude locale portant sur quatre années de statistiques (ANONYME, 1993). Les captures principales de la Saône aval sont : les grands cyprinidés (brème sp., gardon, carpe, chevaine et tanche par ordre d'importance pondérale), les petits cyprinidés (ablette en majorité), le poisson-chat, l'écrevisse américaine, le sandre, le brochet, la perche et le silure.

L'homogénéité d'exploitation a été assurée par la distinction des métiers (CHANGEUX et ZYLBERBLAT, ce numéro) qui est ici combinée aux distinctions sectorielles. Cette régionalisation de la définition du métier permet de réduire la probabilité de s'adresser à plusieurs stocks différents.

Les CPUE ont été calculées pour chaque carnet et chaque stock sur une base mensuelle en ramenant la biomasse au nombre de jours avec captures (en $\mathrm{kg} / \mathrm{jour}$ ).

$$
\text { CPUE }_{i j k}=\frac{\text { Biomasse du stock i pour le mois j du carnet } k}{\text { Nombre de jours avec captures du mois j pour le carnet } k}
$$

Ce calcul conserve toute l'information disponible dans les données, en particulier la variabilité inter-carnets. Pour éviter les cas particuliers, nous avons opéré une sélection préalable des carnets en fonction de leur précision en distinguant les deux catégories de pêcheurs. Chez les professionnels, n'ont été retenus que les métiers pour lesquels, chaque année, un carnet au moins indiquait le nombre de jours de pêche par mois. Chez les amateurs ce nombre minimum de carnets a été fixé à cinq. Les variances de ces CPUE ont été étudiées pour chaque stock en retenant le métier et l'année comme facteurs de variations suivant les méthodes exposées dans SAPORTA (1990).

\section{RÉSULTATS}

\subsection{Captures déclarées}

Au cours des deux années d'étude, il a été déclaré une biomasse totale de $202192 \mathrm{~kg}$ dont une quantité négligeable a fait l'objet d'une estimation à partir du poids moyen de la capture considérée (au total $482 \mathrm{~kg}$ soit seulement $0,2 \%$ ). D'une année sur l'autre, la biomasse d'une catégorie de captures donnée (en rassemblant carnassiers et salmonidés) pour une catégorie de pêcheurs donnée (e.g. les petits cyprinidés capturés par les amateurs) ne change pas de rang (Corrélation des rangs de Spearman, Rho=0,937; $n=12 ; p=0,019)$.

\subsubsection{Répartition temporelle des captures déclarées}

La biomasse totale déclarée par mois (Fig. 1) est minimale en mai, durant la période de fermeture partielle de la pêche. Une fois ramenée à 100 carnets, elle ne varie plus beaucoup au cours de l'année. Seuls l'anguille, l'alose, l'écrevisse, le poisson-chat et les poissons divers (perche-soleil, lamproie marine et espèce non précisée) sont déclarés en proportions importantes durant ce mois.

Cette répartition peut être comparée aux saisons mises en évidence dans l'étude des engins. La période "froide", de janvier à mars, correspond à une basse production marquée 


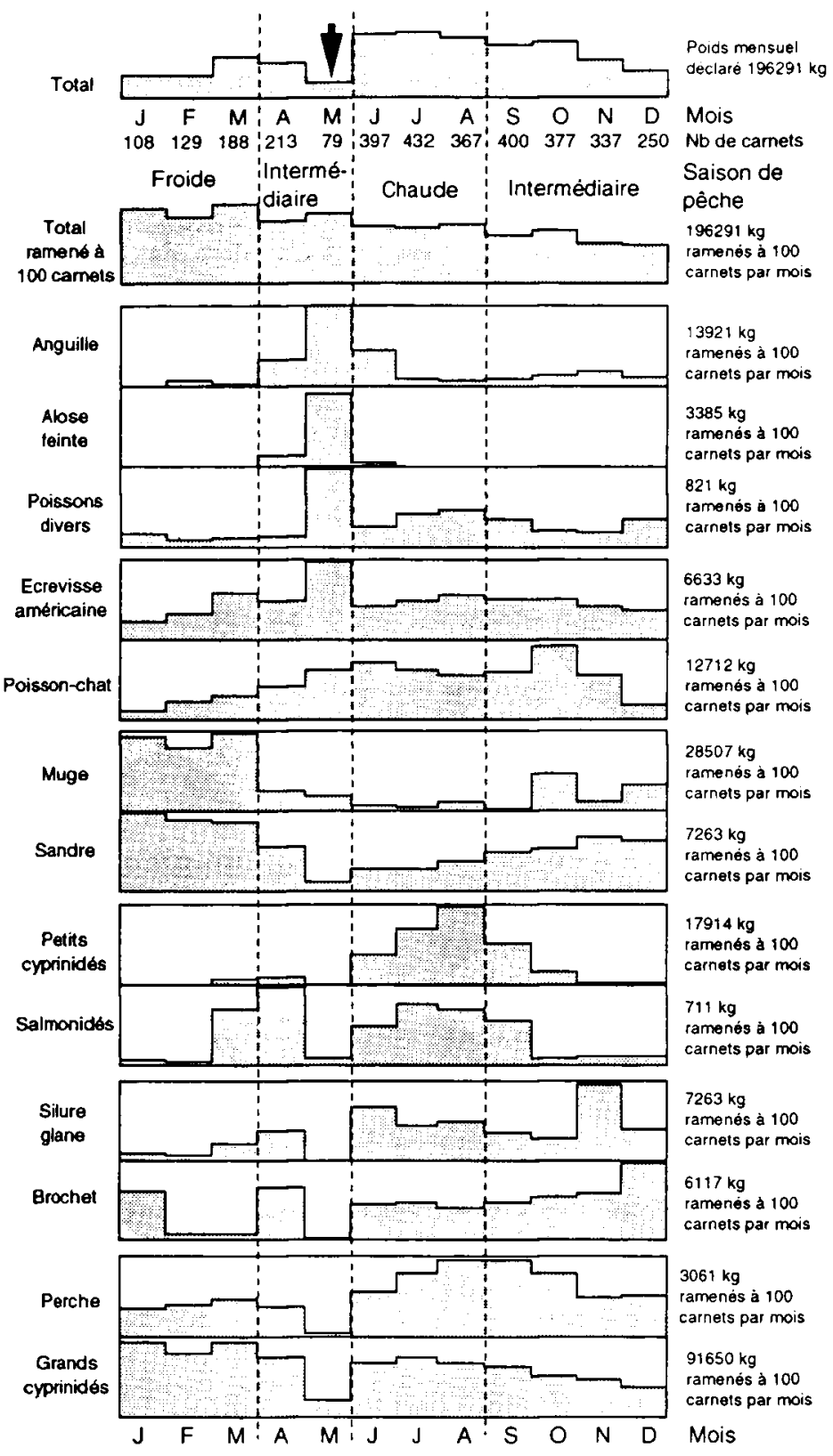

Figure 1 : Distribution mensuelle des biomasses de captures déclarées cumulées sur l'ensemble du domaine d'étude durant les années 1988 et 1989. II n'y a pas d'échelle en ordonnées, car la surface de l'histogramme représente $100 \%$ des captures et la surface d'un élément de l'histogramme (rectangle) représente la fréquence des captures du mois. Les limites saisonnières déduites de l'étude des engins sont indiquées en pointillés. La flèche indique la période de fermeture partielle de la pêche.

Figure 1 : Mensual distribution of the biomass of declared captures, summed over the whole study area for the years 1988 and 1989. There is no scale on the $y$ axis, because the whole surface of the histogram represents $100 \%$ of the captures and the surface of each rectangle of the histogram represents the frequency of the captures in a given month. The fishing seasons, delineated by dashed lines, are given from the preliminary study of fishing gears. The arrow indicates the partial closure of the fishery. 
par la capture de muges, de sandres et de grands cyprinidés. La période "chaude", de juin à septembre, correspond à une haute production marquée par la capture de petits cyprinidés et de salmonidés. La période "intermédiaire" de refroidissement, de septembre à décembre, correspond à une production décroissante au cours de laquelle la proportion de brochets augmente alors que celles de la plupart des autres captures restent stables ou décroissent. Au sein de la période "intermédiaire" de réchauffement, le mois de mai se distingue du mois d'avril par la capture d'une forte proportion d'anguilles, d'aloses, d'écrevisses, de poissonschats et de poissons divers. Pendant ce mois, la pêche est fermée sauf pour espèces migratrices amphihalines ou susceptibles de provoquer des déséquilibres biologiques selon les décrets d'application de l'article 413 du Code rural (poisson-chat, écrevisse américaine, perche-soleil...).

\subsubsection{Répartition sectorielle des captures déclarées}

L'AFC et la classification hiérarchique du tableau de répartition des déclarations entre secteurs ramenées à 100 carnets distinguent le Rhône deltaïque et rassemblent la Saône amont et aval, ainsi que le Rhône amont et aval (Fig. 2).

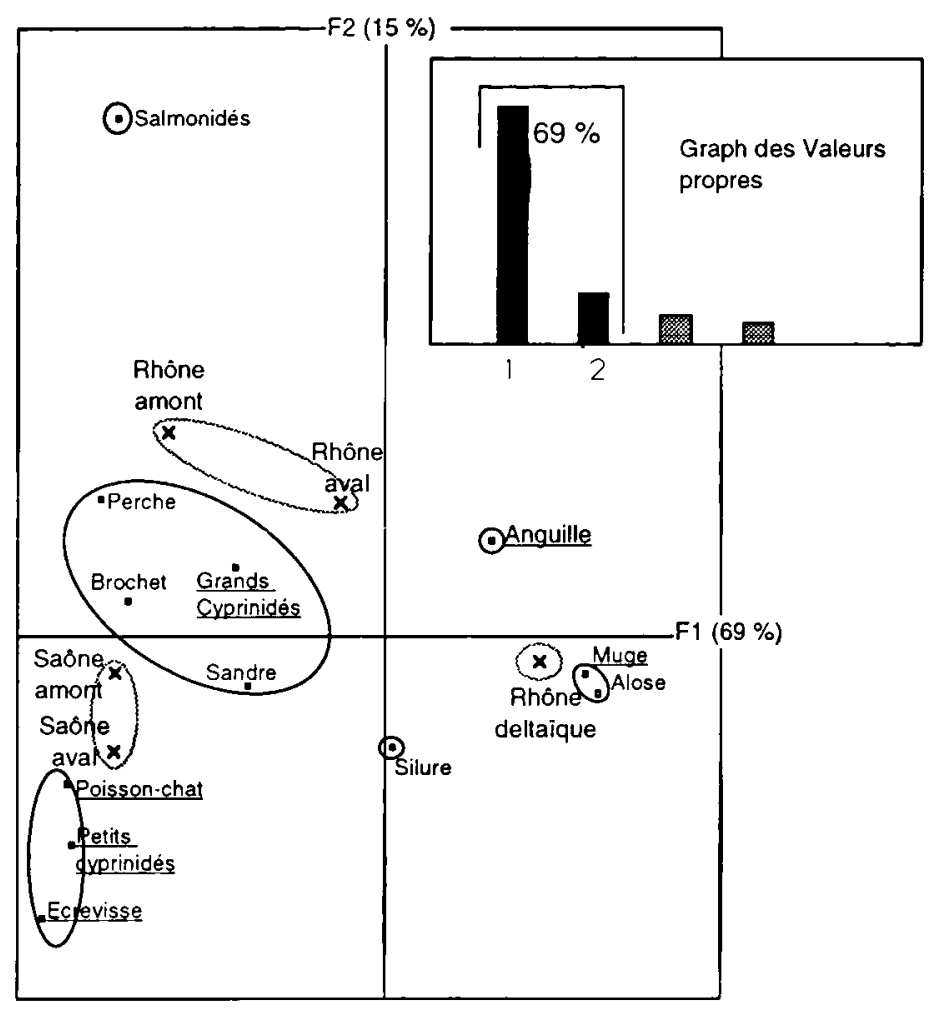

Figure 2 : Plan factoriel F1 x F2 des colonnes (secteurs d'exploitation) et des lignes (captures) de l'AFC de la répartition spatiale des captures. Les groupes entourés sont obtenus par classification hiérarchique. Le pourcentage d'inertie est précisé pour chaque axe. Les captures structurantes (contribuant à plus de $10 \%$ de l'inertie du plan) sont soulignées.

Figure 2 : Factorial map F1 $x$ F2 with columns (exploitation sectors) and lines (captures) of the Correspondance Analysis for the spatial distribution of fish captures. The encircled groups are obtained by hierarchical classification. The percent inertia is given for each axis. Structuring captures (having more than $10 \%$ of relative contribution to the inertia of the map) are underlined. 
Les captures structurantes du plan F1 x F2 sont celles du muge associé au Rhône deltaïque, du poisson-chat, des petits cyprinidés et de l'écrevisse associés au groupe de la Saône amont et aval. Les autres captures structurantes sont celles de l'anguille en position intermédiaire entre le Rhône aval et le Rhône deltaïque, et des grands cyprinidés entre le groupe de la Saône et le groupe du Rhône amont et aval. Les salmonidés se distinguent par une forte association avec le Rhône amont, tandis que le silure se place en position intermédiaire entre le groupe de la Saône et le Rhône deltaïque.

\subsection{Captures estimées}

En 1988 et en 1989, les biomasses totales estimées des prélèvements par la pêche aux engins sont respectivement de $238 \mathrm{t}$ et $273 \mathrm{t}$, toutes catégories de pêcheurs confondues. Les variations inter-annuelles de la composition des captures sont faibles. L'analyse de la variance des biomasses estimées des 12 captures les plus abondantes ne fait ressortir aucun effet significatif de l'année ( $F<1$ chez les amateurs comme chez les professionnels). De même, lorsqu'on distingue les secteurs, aucune partition des estimations ne fait ressortir une influence de l'année en dessous du seuil alpha de $15 \%$. Dans un secteur d'exploitation donné, les corrélations inter-annuelles des estimations sur les douze captures les plus abondantes sont significatives au risque alpha inférieur à $1 \%$ pour les deux catégories de pêcheurs. Ces différentes remarques justifient le calcul des moyennes sur les deux années d'exploitation pour chaque catégorie de pêcheurs et chaque secteur.

\subsubsection{Pêche professionnelle}

Une fois exclus les carnets sans captures, la biomasse déclarée par carnet de pêcheur professionnel varie d'un secteur à l'autre. Les tests de Mann et Whitney menés deux à deux au risque alpha de $5 \%$ montrent que les carnets issus des secteurs du Rhône deltaïque et du Rhône aval présentent une biomasse plus importante (médiane = $2435,8 \mathrm{~kg} /$ carnet) que les carnets des secteurs de la Saône amont et du Rhône amont (médiane $=630,2 \mathrm{~kg} / \mathrm{carnet}$ ) tandis que les carnets de la Saône aval se situent à un niveau intermédiaire (médiane $=1366,5 \mathrm{~kg} /$ carnet).

La biomasse totale estimée des captures effectuées par l'ensemble des professionnels sur le Rhône et la Saône s'élève, en moyenne sur deux ans, à 185,2 tan (Tableau II). Elle est comprise entre 97,4 et 401,3 t/an au risque alpha de $5 \%$. La précision de ce chiffre est assez faible en raison d'une erreur relative importante sur les catégories les plus abondantes (anguille, grands cyprinidés). En revanche, les biomasses de certaines espèces comme la tanche, le brochet, la perche et le sandre sont connues avec une erreur relative inférieure à $60 \%$.

Les grands cyprinidés forment la catégorie de captures la plus importante $(40,4 \%$ du total). Viennent, ensuite, les autres amphihalins $(21,9 \%)$, constitués essentiellement de muges. Les catégories suivantes sont, par ordre d'importance décroissante : l'anguille $(15,2 \%)$, les petits cyprinidés $(9,1 \%)$, les autres captures $(7,9 \%)$ et les carnassiers ajoutés aux salmonidés $(5,7 \%)$. Dans ce dernier groupe, le sandre dépasse le brochet d'environ une tonne.

Certaines captures sont particulièrement abondantes à elles seules sans considérer les catégories. Par exemple, avec 38,7 t/an, le muge représente $20,9 \%$ du total des captures, suivi de l'anguille $(15,2 \%)$, de la brème sp. $(9,5 \%)$ et des cyprinidés sans précision d'espèce $(8,9 \%)$.

La moyenne des captures annuelles estimées par secteur est connue avec une erreur relative comprise entre $28 \%$ et $1900 \%$ (Tableau III). Cette dernière valeur exceptionnelle est atteinte dans le Rhône deltaïque en raison d'une importante variabilité inter-carnets de la biomasse d'anguilles déclarées.

La biomasse totale des captures estimée par secteur varie d'un facteur 28 (de 2,6 à 72,2 t/an). Les différences inter-sectorielles sont donc importantes.

La Saône aval est le premier secteur de production avec une biomasse composée en majorité de grands cyprinidés $(37,6 \mathrm{t} / \mathrm{an})$ et de près d'un quart de petits cyprinidés $(16,6 \mathrm{t} / \mathrm{an})$. La biomasse des carnassiers, ajoutée à celle des salmonidés $(5,6 \mathrm{t}$ /an) est dominée par celle du sandre ( $3,2 \mathrm{t} / \mathrm{an})$. La catégorie des autres captures (12,3 t/an) est surtout composée d'écrevisses américaines (5,3 t/an) et de poissons-chats (5,3 taan). 
Tableau II : Biomasses décarées et estimées (en $\mathrm{kg} / \mathrm{an}$ ) des captures des pêcheurs professionnels sur l'ensemble du domaine d'étude pour les deux années d'exploitation. IC : intervalles de confiance au risque alpha de $5 \%$. * : erreur relative inférieure à $60 \%$.

Table II : Declared and estimated biomass (in $\mathrm{kg} / \mathrm{year}$ ) for captures by professionals (commercial fishermen) on the whole of the study area for the two years of exploitation. IC : confidence intervals (alpha=0.05). * : relative error less than $60 \%$.

\begin{tabular}{|c|c|c|c|c|c|c|}
\hline \multirow{3}{*}{ Anguille } & \multicolumn{2}{|c|}{1988} & \multicolumn{2}{|c|}{1989} & \multicolumn{2}{|c|}{ Moyenne } \\
\hline & \multicolumn{2}{|c|}{ Déclaration Estimation } & \multicolumn{2}{|c|}{ Déclaration Estimation } & \multirow{2}{*}{$\begin{array}{r}\text { Estimation } \\
28210\end{array}$} & \multirow{2}{*}{$\frac{\text { IC }}{\text { ]9818;126243[ }}$} \\
\hline & 5815 & 29915 & 7257 & 26506 & & \\
\hline $\begin{array}{l}\text { Alose feinte } \\
\text { Muge sp. } \\
\text { Bar commun }\end{array}$ & $\begin{array}{r}762 \\
8581 \\
88\end{array}$ & $\begin{array}{r}1067 \\
21114 \\
123\end{array}$ & $\begin{array}{r}998 \\
20813 \\
16\end{array}$ & $\begin{array}{r}2540 \\
56319 \\
41\end{array}$ & $\begin{array}{r}1803 \\
38716 \\
82\end{array}$ & $\begin{array}{l}\text { ]1146;2461[ } \\
\text { ]26390;52004[ } \\
\text { ]70;94[" }\end{array}$ \\
\hline Autres amphihalins & 9431 & 22304 & 21828 & 58900 & 40602 & ]27606;54559[ \\
\hline $\begin{array}{l}\text { Brème sp. } \\
\text { Gardon } \\
\text { Hotu } \\
\text { Carpe ss précision } \\
\text { Chevaine } \\
\text { Carpe commune } \\
\text { Cyprinidés ss précision } \\
\text { Barbeau fluviatile } \\
\text { Tanche } \\
\text { Rotengle }\end{array}$ & $\begin{array}{r}9763 \\
4880 \\
3268 \\
3011 \\
1880 \\
0 \\
4303 \\
2125 \\
1327 \\
323 \\
\end{array}$ & $\begin{array}{r}25450 \\
12616 \\
13582 \\
7788 \\
5179 \\
- \\
9756 \\
8742 \\
3847 \\
709 \\
\end{array}$ & $\begin{array}{r}3863 \\
2841 \\
1944 \\
1957 \\
1182 \\
15 \\
11620 \\
1405 \\
825 \\
165 \\
\end{array}$ & $\begin{array}{r}9788 \\
7201 \\
6766 \\
4134 \\
3631 \\
28 \\
23260 \\
5025 \\
1718 \\
435 \\
\end{array}$ & $\begin{array}{r}17619 \\
9909 \\
10174 \\
5961 \\
4405 \\
14 \\
16508 \\
6884 \\
2782 \\
572 \\
\end{array}$ & $\begin{array}{l}\text { ]8495;49626[ } \\
\text { ]5150;17596[ } \\
\text { ]2605;33533[ } \\
\text { ]3083;8840[ } \\
\text { ]2785;6026[ } \\
\text { ]7;27[ } \\
\text { ]7961;28651[ } \\
\text { ]1908;13613[ } \\
\text { ]2005;3561[ [* } \\
\text { ]401;743[ [ } \\
\end{array}$ \\
\hline Grands Cyprinidés & 30878 & 87670 & 25816 & 61986 & 74828 & ] $34400 ; 162216[$ \\
\hline $\begin{array}{l}\text { Friture ss précision } \\
\text { Ablette } \\
\text { Goujon } \\
\text { Bouvière }\end{array}$ & $\begin{array}{r}2803 \\
1969 \\
12 \\
<1\end{array}$ & $\begin{array}{r}6147 \\
4307 \\
50 \\
1\end{array}$ & $\begin{array}{r}5847 \\
6697 \\
0 \\
0\end{array}$ & $\begin{array}{r}10772 \\
12344 \\
- \\
-\end{array}$ & $\begin{array}{r}8459 \\
8326 \\
25 \\
<1\end{array}$ & $\begin{array}{l}\text { ]4753;12639[ } \\
\text { ]5394;11258[ } \\
\text { ]24;27[** } \\
\text { ]0;2[ }\end{array}$ \\
\hline Petits Cyprinidés & 4784 & 10505 & 12544 & 23116 & 16810 & ]10171;23926[ \\
\hline $\begin{array}{l}\text { Brochet } \\
\text { Perche ss précision } \\
\text { Sandre } \\
\text { Black-bass } \\
\text { Ombre } \\
\text { Truite } \\
\text { Salmonidés ss précision }\end{array}$ & $\begin{array}{r}1586 \\
414 \\
1496 \\
14 \\
13 \\
169 \\
14 \\
\end{array}$ & $\begin{array}{r}4601 \\
1373 \\
3899 \\
30 \\
53 \\
707 \\
60 \\
\end{array}$ & $\begin{array}{r}1228 \\
265 \\
2700 \\
11 \\
6 \\
120 \\
1 \\
\end{array}$ & $\begin{array}{r}2731 \\
681 \\
5540 \\
21 \\
20 \\
433 \\
4 \\
\end{array}$ & $\begin{array}{r}3666 \\
1027 \\
4720 \\
25 \\
36 \\
570 \\
32 \\
\end{array}$ & 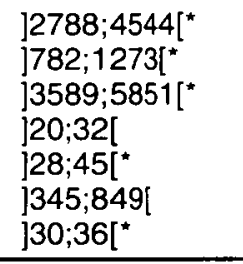 \\
\hline Carnassiers et Salmonidés & 3706 & 10723 & 4330 & 9429 & 10076 & ]7582;12630[ \\
\hline $\begin{array}{l}\text { Ecrevisse américaine } \\
\text { Poisson-chat } \\
\text { Silure glane } \\
\text { Espèce non précisée } \\
\text { Lote }\end{array}$ & $\begin{array}{r}1657 \\
3265 \\
776 \\
4 \\
35 \\
\end{array}$ & $\begin{array}{r}3676 \\
7382 \\
1712 \\
16 \\
129 \\
\end{array}$ & $\begin{array}{r}3887 \\
2943 \\
1675 \\
0 \\
12 \\
\end{array}$ & $\begin{array}{l}7199 \\
5471 \\
3641\end{array}$ & $\begin{array}{r}5438 \\
6427 \\
2677 \\
8 \\
80 \\
\end{array}$ & $\begin{array}{l}\text { ]2936;8129[ } \\
] 3104 ; 9902[ \\
] 1748 ; 3606[ \\
17 ; 9\left[^{*}\right. \\
] 68 ; 95\left[^{\star}\right.\end{array}$ \\
\hline Autres captures & 5737 & 12916 & 8517 & 16343 & 14629 & ] $7863 ; 21741[$ \\
\hline lota & 60351 & 174033 & 80292 & 196280 & 185156 & ]97440;401315[ \\
\hline
\end{tabular}

* erreur relative $<60 \%$ 
Tableau III : Biomasses moyennes estimées (en $\mathrm{kg} / \mathrm{an}$ ) des captures des pêcheurs professionnels suivant le secteur d'exploitation. L'erreur relative au risque alpha de $5 \%$ est précisée pour le total par secteur.

Table III : Estimated average biomass of the captures by professionals (in $\mathrm{kg} / \mathrm{year}$ ) for each sector of exploitation. The relative error (alpha $=0.05$ ) is given for the total biomass.

\begin{tabular}{|c|c|c|c|c|c|c|}
\hline & $\begin{array}{l}\text { Rhône } \\
\text { amont }\end{array}$ & $\begin{array}{l}\text { Rhône } \\
\text { aval }\end{array}$ & $\begin{array}{c}\text { Rhône } \\
\text { deltaïque }\end{array}$ & $\begin{array}{l}\text { Saône } \\
\text { amont }\end{array}$ & $\begin{array}{c}\text { Saône } \\
\text { aval }\end{array}$ & Total \\
\hline Anguille & 37 & 22584 & 5383 & 2 & 205 & 28210 \\
\hline $\begin{array}{l}\text { Alose feinte } \\
\text { Muge sp. } \\
\text { Bar commun }\end{array}$ & $\begin{array}{l}- \\
-\end{array}$ & $\begin{array}{r}48 \\
10265\end{array}$ & $\begin{array}{r}1756 \\
28451 \\
82\end{array}$ & $\begin{array}{l}- \\
-\end{array}$ & 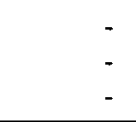 & $\begin{array}{r}1803 \\
38716 \\
82\end{array}$ \\
\hline Autres amphihalins & - & 10313 & 30289 & & - & 40602 \\
\hline $\begin{array}{l}\text { Brème sp. } \\
\text { Gardon } \\
\text { Hotu } \\
\text { Carpe ss précision } \\
\text { Chevaine } \\
\text { Carpe commune } \\
\text { Cyprinidés ss précision } \\
\text { Barbeau fluviatile } \\
\text { Tanche } \\
\text { Rotengle }\end{array}$ & $\begin{array}{r}671 \\
1526 \\
8519 \\
200 \\
1980 \\
- \\
1020 \\
4294 \\
1021 \\
29\end{array}$ & $\begin{array}{r}4932 \\
1549 \\
1151 \\
1093 \\
511 \\
4 \\
475 \\
1889 \\
116 \\
86 \\
\end{array}$ & $\begin{array}{r}389 \\
1065 \\
139 \\
363 \\
42 \\
- \\
2894 \\
86 \\
-\end{array}$ & $\begin{array}{r}81 \\
155 \\
61 \\
255 \\
39 \\
- \\
522 \\
17 \\
114 \\
-\end{array}$ & $\begin{array}{r}11546 \\
5614 \\
303 \\
4051 \\
1833 \\
14 \\
11598 \\
598 \\
1531 \\
457\end{array}$ & $\begin{array}{r}17619 \\
9909 \\
10174 \\
5961 \\
4405 \\
14 \\
16508 \\
6884 \\
2782 \\
572\end{array}$ \\
\hline Grands Cyprinidés & 19261 & 11801 & 4978 & 1243 & 37545 & 74828 \\
\hline $\begin{array}{l}\text { Friture ss précision } \\
\text { Ablette } \\
\text { Goujon } \\
\text { Bouvière } \\
\end{array}$ & $\begin{array}{r}- \\
- \\
-\end{array}$ & . & $\begin{array}{l}- \\
- \\
- \\
-\end{array}$ & $\begin{array}{r}126 \\
53 \\
- \\
\end{array}$ & $\begin{array}{r}8333 \\
8273 \\
- \\
<1 \\
\end{array}$ & $\begin{array}{r}8459 \\
8326 \\
25 \\
<1 \\
\end{array}$ \\
\hline Petits Cyprinidés & 25 & - & - & 179 & 16606 & 16810 \\
\hline $\begin{array}{l}\text { Brochet } \\
\text { Perche ss précision } \\
\text { Sandre } \\
\text { Black-bass } \\
\text { Ombre } \\
\text { Truite } \\
\text { Salmonidés ss précision }\end{array}$ & $\begin{array}{r}1496 \\
665 \\
12 \\
- \\
36 \\
569 \\
32 \\
\end{array}$ & $\begin{array}{r}84 \\
17 \\
864 \\
: \\
: \\
: \\
\end{array}$ & $\begin{array}{r}83 \\
- \\
464 \\
- \\
- \\
-\end{array}$ & $\begin{array}{r}46 \\
7 \\
147 \\
4 \\
- \\
- \\
-\end{array}$ & $\begin{array}{r}1957 \\
339 \\
3233 \\
22 \\
- \\
1 \\
\end{array}$ & $\begin{array}{r}3666 \\
1027 \\
4720 \\
25 \\
36 \\
570 \\
32 \\
\end{array}$ \\
\hline Carnassiers et Salmonidés & 2809 & 965 & 547 & 203 & 5552 & 10076 \\
\hline $\begin{array}{l}\text { Ecrevisse américaine } \\
\text { Poisson-chat } \\
\text { Silure glane } \\
\text { Espèce non précisée } \\
\text { Lote }\end{array}$ & $\begin{array}{r}93 \\
169 \\
- \\
8 \\
66\end{array}$ & $\begin{array}{r}: \\
102 \\
:\end{array}$ & $\begin{array}{r}- \\
899 \\
- \\
-\end{array}$ & $\begin{array}{r}- \\
1002 \\
- \\
- \\
<1\end{array}$ & $\begin{array}{r}5345 \\
5255 \\
1675 \\
- \\
13\end{array}$ & $\begin{array}{r}5438 \\
6427 \\
2677 \\
8 \\
80\end{array}$ \\
\hline Autres captures & 336 & 102 & 899 & 1003 & 12289 & 14629 \\
\hline $\begin{array}{l}\text { Total } \\
\text { Erreur relative* }\end{array}$ & $\begin{array}{l}22468 \\
38 \%\end{array}$ & $\begin{array}{l}45765 \\
28 \%\end{array}$ & $\begin{array}{r}42096 \\
1900 \%\end{array}$ & $\begin{array}{r}2630 \\
51 \%\end{array}$ & $\begin{array}{l}72196 \\
25 \%\end{array}$ & 185156 \\
\hline
\end{tabular}

* au risque alpha de $5 \%$; - déclaration nulle 
Les estimations totales du Rhône aval et du Rhône deltaïque sont toutes deux situées aux alentours de 44 tan, mais leurs compositions sont relativement différentes l'une de l'autre bien qu'elles aient en commun la présence de poissons d'origine marine. Les captures du Rhône aval présentent une forte quantité d'anguilles $(22,6 \mathrm{t} / \mathrm{an})$, tandis que celles du Rhône deltaïque sont dominées par les autres amphihalins avec, en particulier, 30,3 tan de muges.

La biomasse totale estimée des captures du Rhône amont ne représente plus que la moitié des estimations des secteurs précédents. Elle est dominée par la catégorie des grands cyprinidés $(19,3 \mathrm{t} / \mathrm{an})$, composée pour moitié de hotus ( $8,5 \mathrm{t} / \mathrm{an})$ alors qu'aucun autre secteur ne dépasse 2 tan pour cette espèce. C'est également dans ce secteur que les biomasses de salmonidés sont maximales sur le bassin $(0,6 \mathrm{t} / \mathrm{an})$. Parmi les carnassiers, les quantités de brochet dominent ici nettement celles de sandre $(1,5 \mathrm{t} / \mathrm{an}$ contre $12 \mathrm{~kg} / \mathrm{an}$ respectivement).

Enfin, la Saône amont, apparaît comme un secteur d'exploitation où les captures des pêcheurs professionnels sont particulièrement faibles.

\subsubsection{Pêche amateur}

Une fois exclus les carnets sans captures, la biomasse déclarée par carnet de pêcheur amateur présente une médiane nettement plus faible que celle des professionnels $(75,95 \mathrm{~kg} /$ carnet $)$.

La biomasse totale estimée des captures effectuées par l'ensemble de ces pêcheurs sur le Rhône et la Saône s'élève, en moyenne sur deux ans, à 71,0 t/an (Tableau IV). Elle est comprise entre 48,9 et 94,2 tan au risque alpha de $5 \%$. Cette estimation est plus précise que la précédente en raison d'une faible erreur relative sur la majorité des grands cyprinidés.

Les grands cyprinidés forment la catégorie de captures la plus abondante $(56,3 \% \mathrm{du}$ total). Les catégories suivantes sont les autres captures $(14,9 \%)$, avec une majorité de poissons-chats, les carnassiers ajoutés aux salmonidés $(13,8 \%)$, ou le brochet et le sandre sont à parts égales, puis la catégorie des autres amphihalins, dominée par l'alose feinte. Les petits cyprinidés et l'anguille sont des catégories peu importantes (respectivement 3,9 et $1,9 \%$ du total). La brème sp., capture la plus abondante avec 6,2 tan, fait $11,2 \%$ de la biomasse estimée pour les amateurs. En dehors de certaines espèces de grands cyprinidés, comme le hotu ou le gardon, le poisson-chat est la seule espèce à dépasser 5 tan .

L'erreur relative sur la moyenne de la biomasse totale des captures annuelles estimées par secteur est comprise entre 9 et $44 \%$ (Tableau V). La meilleure précision est obtenue en Saône aval, où les captures sont également les plus abondantes. La moins bonne est atteinte dans Rhône deltaïque en raison d'un taux de retours particulièrement faible des carnets.

Le total des captures estimées varie à peu près du simple au double entre les secteurs.

Avec 18,9 tan, la Saône aval est le premier secteur de production. Les catégories les plus importantes sont les grands cyprinidés $(8,0 \mathrm{t} / \mathrm{an})$, dominés par la brème $\mathrm{sp}$. $(2,2 \mathrm{t} / \mathrm{an})$, et les autres captures $(6,2 \mathrm{t} / \mathrm{an})$ avec le poisson-chat $(3,4 \mathrm{t} / \mathrm{an})$, l'écrevisse américaine $(1,5 \mathrm{Van})$ et le silure glane $(1,1 \mathrm{Van})$. La catégorie des carnassiers ajoutée aux salmonidés $(3,6 \mathrm{t} / \mathrm{an})$ est dominée par le brochet et le sandre qui font chacun environ 1,3 tan. Avec moins d'une tonne par an, les petits cyprinidés ne forment que $5 \%$ des captures totales estimées du secteur pour les amateurs.

La Saône amont présente une composition des captures relativement proche de la précédente pour des quantités bien moindres (12,4 tan au total).

En revanche, la composition des captures des secteurs du Rhône amont et aval est nettement différente de celle des secteurs précédents, avec toujours environ trois quarts de grands cyprinidés, et une majorité de carnassiers et salmonidés dans le quart restant.

Dans le Rhône amont, les 10,8 tan de grands cyprinidés capturés sont composées pour un tiers de hotus $(3,4 \mathrm{t} / \mathrm{an})$ et un quart de barbeaux fluviatiles $(2,4 \mathrm{t} / \mathrm{an})$. La perche et le brochet dominent nettement dans les 2,2 tan de carnassiers et de salmonidés capturés 
Tableau IV : Biomasses déclarées et estimées (en $\mathrm{kg} / \mathrm{an}$ ) des captures des pêcheurs amateurs sur l'ensemble du domaine d'étude pour les deux années d'exploitation. IC : intervalles de confiance au risque alpha de $5 \%$. ${ }^{\star}$ : erreur relative inférieure à $60 \%$.

Table IV : Declared and estimated biomass (in $\mathrm{kg} / \mathrm{year}$ ) for captures by amateurs (recreational fishermen) on the whole of the study area for the two years of exploitation. IC : confidence intervals (alpha $=0.05$ ). ${ }^{*}$ : relative error less than $60 \%$.

\begin{tabular}{|c|c|c|c|c|c|c|}
\hline \multirow[b]{3}{*}{ Anguille } & \multicolumn{2}{|c|}{1988} & \multicolumn{2}{|c|}{1989} & \multicolumn{2}{|c|}{ Moyenne } \\
\hline & Déclaration & Estimation & Déclaration & Estimation & Estimation & IC \\
\hline & 429 & 1434 & 435 & 1322 & 1378 & ]507;2332[ \\
\hline $\begin{array}{l}\text { Alose feinte } \\
\text { Muge sp. } \\
\text { Lamproie marine } \\
\text { Bar commun }\end{array}$ & $\begin{array}{r}657 \\
405 \\
35 \\
28\end{array}$ & $\begin{array}{r}3850 \\
2341 \\
208 \\
166\end{array}$ & $\begin{array}{r}968 \\
212 \\
1 \\
5\end{array}$ & $\begin{array}{r}5150 \\
1100 \\
6 \\
29\end{array}$ & $\begin{array}{r}4500 \\
1720 \\
107 \\
98\end{array}$ & $\begin{array}{l}\text { ]1313;7893[ } \\
\text { ]622;2819[ } \\
\text { ]17;238[ } \\
\text { ]16;234[ }\end{array}$ \\
\hline Autres amphihalins & 1124 & 6565 & 1187 & 6285 & 6425 & ]1968;11184[ \\
\hline $\begin{array}{l}\text { Brème sp. } \\
\text { Gardon } \\
\text { Hotu } \\
\text { Carpe ss précision } \\
\text { Chevaine } \\
\text { Cyprinidés ss précision } \\
\text { Barbeau fluviatile } \\
\text { Tanche } \\
\text { Rotengle } \\
\text { Vandoise }\end{array}$ & $\begin{array}{r}2376 \\
1778 \\
2324 \\
1303 \\
933 \\
3616 \\
1469 \\
1246 \\
156 \\
95 \\
\end{array}$ & $\begin{array}{r}6042 \\
3874 \\
4631 \\
2772 \\
2178 \\
8396 \\
3069 \\
3848 \\
312 \\
172 \\
\end{array}$ & $\begin{array}{r}4486 \\
2994 \\
3425 \\
1685 \\
2042 \\
1176 \\
2118 \\
1348 \\
638 \\
45 \\
\end{array}$ & $\begin{array}{r}9936 \\
6633 \\
7782 \\
3579 \\
4888 \\
2385 \\
4908 \\
2963 \\
1603 \\
73 \\
\end{array}$ & $\begin{array}{r}7989 \\
5253 \\
6207 \\
3176 \\
3533 \\
5390 \\
3988 \\
3405 \\
958 \\
123 \\
\end{array}$ & 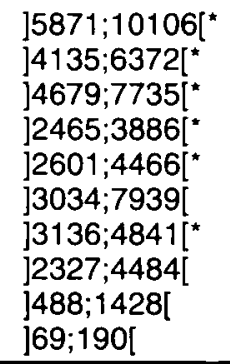 \\
\hline Grands cyprinidés & 15295 & 35293 & 19957 & 44750 & 40021 & ]28805;51447[ \\
\hline $\begin{array}{l}\text { Friture } \\
\text { Ablette } \\
\text { Spirlin } \\
\text { Goujon } \\
\end{array}$ & $\begin{array}{r}233 \\
663 \\
2 \\
8 \\
\end{array}$ & $\begin{array}{r}968 \\
1549 \\
3 \\
16 \\
\end{array}$ & $\begin{array}{r}535 \\
991 \\
0 \\
55 \\
\end{array}$ & $\begin{array}{r}970 \\
1924 \\
. \\
97 \\
\end{array}$ & $\begin{array}{r}969 \\
1737 \\
1 \\
57 \\
\end{array}$ & $\begin{array}{l}\text { ]448;1739[ } \\
\text { ]1202;2272[ } \\
\text { ]1;4[ } \\
] 31 ; 90[\end{array}$ \\
\hline Petits cyprinidés & 906 & 2536 & 1581 & 2992 & 2764 & ]1681;4105[ \\
\hline $\begin{array}{l}\text { Brochet } \\
\text { Perche ss précision } \\
\text { Sandre } \\
\text { Black-bass } \\
\text { Ombre } \\
\text { Truite } \\
\text { Salmonidés ss précision }\end{array}$ & $\begin{array}{r}1428 \\
1196 \\
1279 \\
60 \\
20 \\
171 \\
2 \\
\end{array}$ & $\begin{array}{r}3178 \\
2199 \\
3209 \\
142 \\
38 \\
335 \\
3 \\
\end{array}$ & $\begin{array}{r}1990 \\
1249 \\
1901 \\
27 \\
22 \\
172 \\
1 \\
\end{array}$ & $\begin{array}{r}3843 \\
2308 \\
3953 \\
77 \\
46 \\
340 \\
2 \\
\end{array}$ & $\begin{array}{r}3510 \\
2253 \\
3581 \\
110 \\
42 \\
338 \\
3 \\
\end{array}$ & $\begin{array}{l}\text { ]2951;:4071["* } \\
\text { ]1868;2638[* } \\
\text { ]2857;4305[* } \\
\text { ]68;152[ } \\
\text { ]21;73[ } \\
\text { ]233;443[ } \\
\text { ]2;6[ } \\
\end{array}$ \\
\hline Carnassiers et Salmonidés & 4156 & 9105 & 5363 & 10569 & 9837 & ]7999;11688[ \\
\hline $\begin{array}{l}\text { Ecrevisse américaine } \\
\text { Poisson-chat } \\
\text { Silure glane } \\
\text { Perche-soleil } \\
\text { Espèce non précisée } \\
\text { Grémille } \\
\text { Lote }\end{array}$ & $\begin{array}{r}817 \\
3325 \\
884 \\
24 \\
32 \\
0 \\
88\end{array}$ & $\begin{array}{r}1679 \\
6380 \\
1479 \\
35 \\
57 \\
- \\
158\end{array}$ & $\begin{array}{r}1433 \\
3208 \\
958 \\
288 \\
0 \\
<1 \\
60\end{array}$ & $\begin{array}{r}2116 \\
7089 \\
1554 \\
422 \\
2 \\
1 \\
131\end{array}$ & $\begin{array}{r}1898 \\
6734 \\
1517 \\
229 \\
30 \\
<1 \\
144\end{array}$ & $\begin{array}{l}\text { ]1293;2681[ } \\
\text { ]5367;;102[" } \\
\text { ]1028;2006[ } \\
\text { ]156;394[ } \\
\text { ]15;67[ } \\
\text { ]0;3[ } \\
\text { ]98;191[ }\end{array}$ \\
\hline Autres captures & 5169 & 9789 & 5948 & 11316 & 10552 & ] $7957 ; 13444[$ \\
\hline Total & 27078 & 64721 & 34471 & 77234 & 70977 & ] $48917 ; 94200[$ \\
\hline
\end{tabular}

* erreur relative $<60 \%$ 
Tableau V : Biomasses moyennes estimées (en $\mathrm{kg} / \mathrm{an}$ ) des captures des pêcheurs amateurs suivant le secteur d'exploitation. L'erreur relative au risque alpha de $5 \%$ est précisée pour le total par secteur.

Table V : Estimated average biomass of the captures by amateurs (in $\mathrm{kg} / \mathrm{year}$ ) for each sector of exploitation. The relative error (alpha=0.05) is given for the total biomass.

\begin{tabular}{|c|c|c|c|c|c|c|}
\hline & $\begin{array}{l}\text { Rhône } \\
\text { amont }\end{array}$ & $\begin{array}{c}\text { Rhône } \\
\text { aval }\end{array}$ & $\begin{array}{c}\text { Rhône } \\
\text { deltaïque }\end{array}$ & $\begin{array}{l}\text { Saône } \\
\text { amont }\end{array}$ & $\begin{array}{c}\text { Saône } \\
\text { aval }\end{array}$ & Total \\
\hline Anguille & 124 & 277 & 698 & 116 & 163 & 1378 \\
\hline $\begin{array}{l}\text { Alose feinte } \\
\text { Muge sp. } \\
\text { Lamproie marine } \\
\text { Bar commun }\end{array}$ & $\begin{array}{l}3 \\
- \\
- \\
-\end{array}$ & $\begin{array}{r}44 \\
55 \\
- \\
-\end{array}$ & $\begin{array}{r}4454 \\
1665 \\
107 \\
98\end{array}$ & $\begin{array}{l}- \\
- \\
-\end{array}$ & $\begin{array}{l}- \\
- \\
- \\
-\end{array}$ & $\begin{array}{r}4500 \\
1720 \\
107 \\
98\end{array}$ \\
\hline Autres amphihalins & 3 & 99 & 6323 & - & - & 6425 \\
\hline $\begin{array}{l}\text { Brème sp. } \\
\text { Gardon } \\
\text { Hotu } \\
\text { Carpe ss précision } \\
\text { Chevaine } \\
\text { Cyprinidés ss précision } \\
\text { Barbeau fluviatile } \\
\text { Tanche } \\
\text { Rotengle } \\
\text { Vandoise }\end{array}$ & $\begin{array}{r}999 \\
1519 \\
3420 \\
118 \\
1115 \\
623 \\
2364 \\
249 \\
330 \\
82\end{array}$ & $\begin{array}{r}2347 \\
1529 \\
2410 \\
535 \\
1351 \\
1230 \\
1083 \\
678 \\
322 \\
6\end{array}$ & $\begin{array}{r}1409 \\
157 \\
217 \\
140 \\
318 \\
349 \\
15 \\
6 \\
-\end{array}$ & $\begin{array}{r}1034 \\
944 \\
72 \\
955 \\
440 \\
1695 \\
123 \\
1576 \\
239 \\
-\end{array}$ & $\begin{array}{r}2199 \\
1105 \\
89 \\
1427 \\
309 \\
1843 \\
69 \\
886 \\
61 \\
35\end{array}$ & $\begin{array}{r}7989 \\
5253 \\
6207 \\
3176 \\
3533 \\
5390 \\
3988 \\
3405 \\
958 \\
123\end{array}$ \\
\hline Grands cyprinidés & 10818 & 11490 & 2611 & 7079 & 8023 & 40021 \\
\hline $\begin{array}{l}\text { Friture } \\
\text { Ablette } \\
\text { Spirlin } \\
\text { Goujon }\end{array}$ & $\begin{array}{r}14 \\
251 \\
1 \\
37\end{array}$ & $\begin{array}{r}175 \\
760 \\
- \\
1\end{array}$ & $\begin{array}{r}- \\
60 \\
- \\
-\end{array}$ & $\begin{array}{r}443 \\
124 \\
- \\
-\end{array}$ & $\begin{array}{r}337 \\
541 \\
- \\
18\end{array}$ & $\begin{array}{r}969 \\
1737 \\
1 \\
57\end{array}$ \\
\hline Petits cyprinidés & 303 & 937 & 60 & 567 & 896 & 2764 \\
\hline $\begin{array}{l}\text { Brochet } \\
\text { Perche ss précision } \\
\text { Sandre } \\
\text { Black-bass } \\
\text { Ombre } \\
\text { Truite } \\
\text { Salmonidés ss précision }\end{array}$ & $\begin{array}{r}793 \\
805 \\
218 \\
6 \\
37 \\
313 \\
3\end{array}$ & $\begin{array}{r}427 \\
228 \\
988 \\
39 \\
5 \\
5\end{array}$ & $\begin{array}{r}108 \\
5 \\
305 \\
6 \\
- \\
7 \\
-\end{array}$ & $\begin{array}{r}811 \\
285 \\
799 \\
33 \\
- \\
4\end{array}$ & $\begin{array}{r}1370 \\
930 \\
1270 \\
26 \\
- \\
9 \\
-\end{array}$ & $\begin{array}{r}3510 \\
2253 \\
3581 \\
110 \\
42 \\
338 \\
3\end{array}$ \\
\hline Carnassiers et Salmonidés & 2175 & 1692 & 432 & 1932 & 3606 & 9837 \\
\hline $\begin{array}{l}\text { Ecrevisse américaine } \\
\text { Poisson-chat } \\
\text { Silure glane } \\
\text { Perche-soleil } \\
\text { Espèce non précisée } \\
\text { Grémille } \\
\text { Lote }\end{array}$ & $\begin{array}{r}4 \\
437 \\
- \\
6 \\
25 \\
<1 \\
115\end{array}$ & $\begin{array}{r}6 \\
304 \\
264 \\
- \\
- \\
- \\
2\end{array}$ & $\begin{array}{r}2 \\
274 \\
146 \\
: \\
: \\
-\end{array}$ & $\begin{array}{r}377 \\
2357 \\
- \\
1 \\
1 \\
- \\
13\end{array}$ & $\begin{array}{r}1508 \\
3361 \\
1106 \\
222 \\
3 \\
- \\
14\end{array}$ & $\begin{array}{r}1898 \\
6734 \\
1517 \\
229 \\
30 \\
<1 \\
144\end{array}$ \\
\hline Autres captures & 588 & 577 & 422 & 2750 & 6215 & 10552 \\
\hline$\overline{\text { Total }}$ & 14013 & 15070 & 10547 & 12444 & 18903 & 70977 \\
\hline Erreur relative* & $16 \%$ & $26 \%$ & $44 \%$ & $37 \%$ & $9 \%$ & \\
\hline
\end{tabular}

*au risque alpha de $5 \%$; - déclaration nulle 
avec environ 0,8 tan chacun alors que le sandre prend une part négligeable des apports des pêcheurs amateurs $(0,2 \mathrm{t} / \mathrm{an})$. C'est le seul secteur où l'on dépasse les $50 \mathrm{~kg}$ de truites en captures totales avec une estimation de 0,3 tan pour cette espèce. La présence d'alose feinte est très peu probable dans le Rhône amont. Elle résulte d'une déclaration erronée effectuée par un seul carnet qui conduit à une biomasse estimée négligeable.

Dans le Rhône aval, les 11,5 t/an de grands cyprinidés sont composées en proportions égales de hotus et de brèmes sp.. Les captures de gardons, avec celles de chevaines et de barbeaux restent aux alentours de 1,5 t/an. Le quart restant est plus diversifié, avec notamment l'apparition de petits cyprinidés et d'anguilles en quantités faibles mais non négligeables $(0,9 \mathrm{t} / \mathrm{an}$ et $0,3 \mathrm{t} / \mathrm{an}$ respectivement). Les $1,7 \mathrm{t} / \mathrm{an} \mathrm{de}$ carnassiers et salmonidés sont composées en majorité de sandres $(1,0$ tan).

Enfin, le Rhône deltaïque est le seul secteur dominé par les poissons d'origine marine euryhalins et amphihalins. Les 0,7 tan d'anguilles représentent un dixième des autres amphihalins (6,3 t/an) parmi lesquels on trouve une majorité d'aloses ( $4,5 \mathrm{t} / \mathrm{an})$. La catégorie des grands cyprinidés $(2,6$ t/an) ne représente plus qu'un quart des captures totales avec une majorité de brème sp. (1,4 tan).

\subsubsection{Comparaison entre pêche amateur et pêche professionnelle}

La biomasse totale des captures des amateurs représente, en moyenne, $28 \%$ du prélèvement total estimé. La comparaison des estimations moyennes par catégories fait ressortir la spécialisation des professionnels pour l'anguille, les autres amphihalins et les petits cyprinidés (Fig. 3). La prépondérance des grands cyprinidés est commune aux deux profils et les quantités de carnassiers ajoutées aux salmonidés ainsi que celles des autres captures sont proches pour les deux catégories de pêcheurs.

Les captures des amateurs sont comparables, voire légèrement supérieures à celles des professionnels, pour certaines catégories de captures (carnassiers et salmonidés, autres captures), certaines espèces (alose, brochet, sandre, poisson-chat dans les Tableaux II et IV) ou bien toutes captures confondues dans un secteur comme la Saône amont (Tableau III et V).

\subsubsection{Comparaison entre les différents secteurs d'exploitation}

Le secteur le plus important pour l'ensemble des pêcheurs aux engins est la Saône aval qui concentre $36 \%$ de la production sur $15 \%$ du linéaire étudié. Sur le bassin rhodanien, le rendement kilométrique, toutes captures confondues, varie entre 44,5 et $558,1 \mathrm{~kg} / \mathrm{km} /$ an suivant les années et les secteurs (la variabilité inter-lots n'est pas retenue dans ce calcul qui ramène la quantité estimée par an au linéaire exploité dans chaque secteur). La part des amateurs est toujours plus faible, sauf dans la Saône amont.

Comme précédemment, l'analyse de la variance du rendement par secteur montre que les deux années sont semblables $(F<1)$; ce qui justifie le calcul des moyennes inter-annuelles (Fig. 4).

Malgré une répartition hétérogène visible, l'effet du secteur sur le rendement n'est significatif, en dessous du risque alpha de $5 \%$, que pour les amateurs. Dans ce cas, les tests $t$ de Student, deux à deux au risque alpha de $5 \%$, permettent de distinguer la Saône aval (où le rendement est plus élevé que partout ailleurs), des secteurs à rendements faibles, constitués dans un ordre d'importance décroissante, du Rhône amont, du Rhône aval et de la Saône amont alors que le Rhône deltaïque se situe en position intermédiaire entre ces deux ensembles.

Le rendement surfacique du Rhône se situe entre 10,3 et $23,5 \mathrm{~kg} / \mathrm{ha} / \mathrm{an}$ suivant les secteurs et les années. L'analyse de sa variance conduit aux mêmes conclusions que celles du rendement kilométrique.

\subsection{Captures par Unité d'Effort}

Dans la Saône aval, 91 carnets amateurs et 6 carnets professionnels satisfont nos conditions de sélection. Les carnets professionnels concernent uniquement des pêcheurs 
utilisant simultanément des filets et des pièges. Les carnets amateurs concernent trois métiers différents.

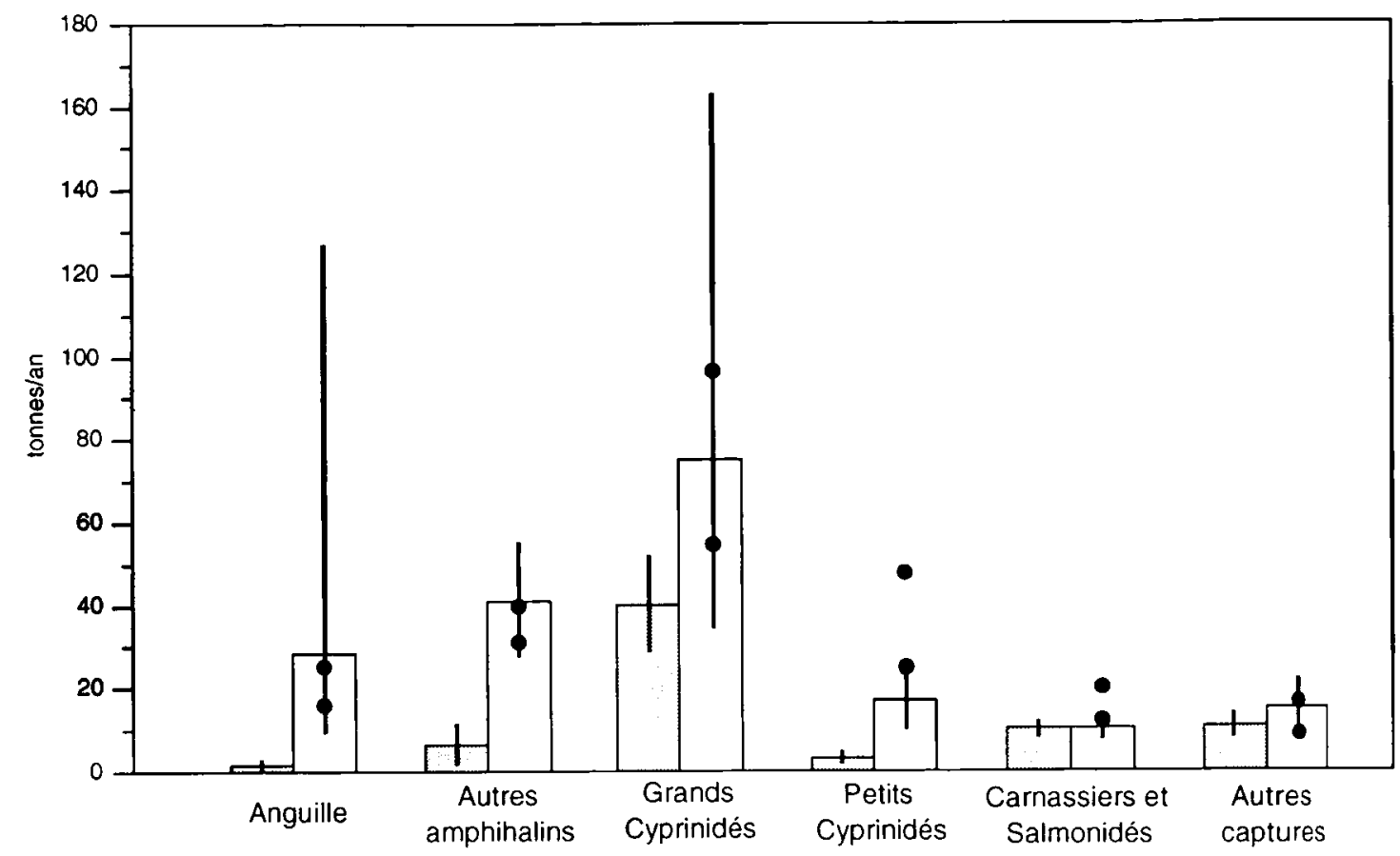

Figure 3 : Biomasse moyenne annuelle estimée des captures chez les amateurs (en gris) et chez les professionnels (en blanc). Les traits verticaux représentent les intervalles de confiance au risque alpha de $5 \%$. Les valeurs de BABIN (1991) sont figurées par des points. Elles correspondent à une hypothèse haute et une hypothèse basse.

Figure 3 : Average annual biomass estimated from the captures of amateurs (grey) and of professionals (white). The vertical lines indicate the confidence intervals at alpha $=0.05$. The results of BABIN (1991) are given as closed circles and correspond to hypothetical upper and lower limits.

Les CPUE des professionnels sont comprises entre 0,011 et $120 \mathrm{~kg} / \mathrm{jour}$ (médiane $=1,7 \mathrm{~kg} / \mathrm{jour}$ ), celles des amateurs sont comprises entre 0,025 et $70 \mathrm{~kg} / \mathrm{jour}$ (médiane $=0,9 \mathrm{~kg} / \mathrm{jour}$ ). Les CPUE des professionnels sont significativement supérieures à celles des amateurs ( $p<0,0001$ au test $U$ de Mann et Whitney).

Une fois transformées par les logarithmes népériens (In), la distribution des $\ln$ (CPUE) se rapproche de la normalité avec une tendance centrale nette qui autorise l'utilisation des méthodes statistiques paramétriques.

\subsubsection{Pêche professionnelle}

Comme un seul métier professionnel répond à nos conditions de sélection, il n'est pas possible d'effectuer de comparaison inter-métiers des CPUE. En revanche, il est possible d'effectuer une comparaison inter-annuelle des CPUE sur tous les stocks sélectionnés.

Les seules variations significatives, inférieures au risque alpha de $5 \%$, concernent le poisson-chat et les grands cyprinidés qui diminuent de 1988 à 1989 (Tableau VI).

\subsubsection{Pêche amateur}

Les trois métiers amateurs offrant la possibilité d'une comparaison inter-métiers et inter-annuelle des $\ln$ (CPUE) sont les pêcheurs aux carrelets, les pêcheurs aux pièges et les pêcheurs utilisant des carrelets et des pièges simultanément au cours de leur année de 
pêche. Dans l'analyse de la variance à deux facteurs (métiers et années) des In(CPUE), l'effet métier est souvent significatif au risque alpha inférieur à $5 \%$, alors que l'effet année agit rarement avec la même signification (Tableau VII). Un effet d'interaction important signale que les $\ln (C P U E)$ ne varient pas de la même manière selon les métiers entre les deux années.

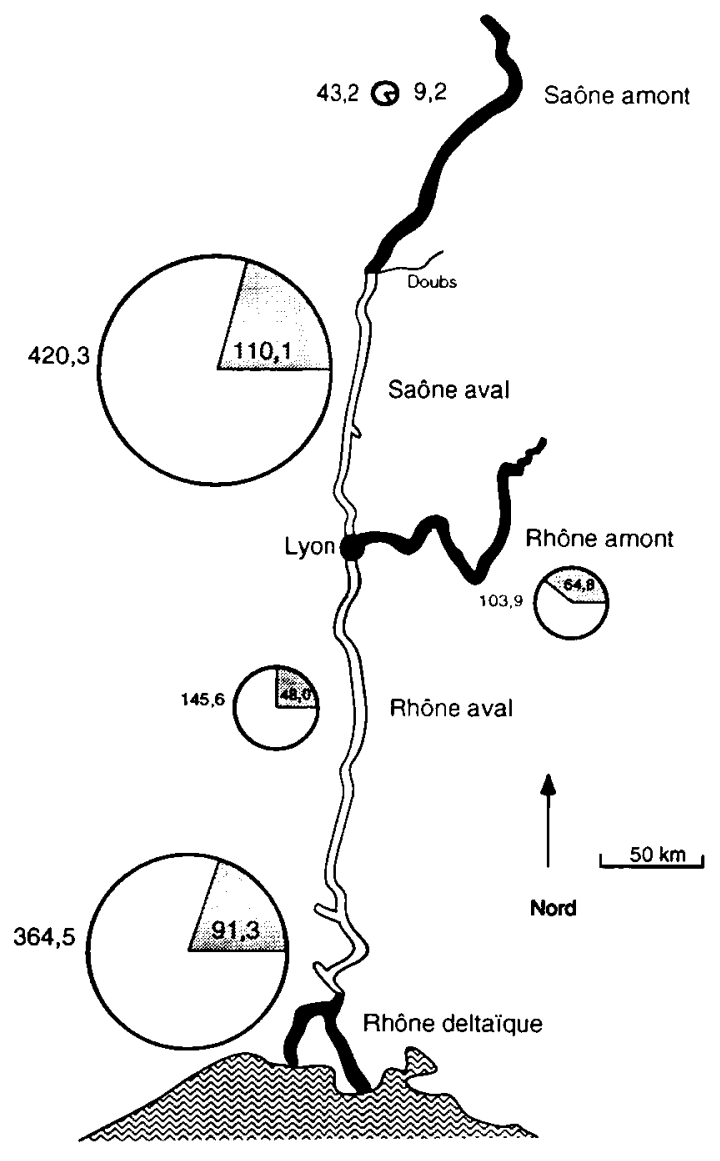

Figure 4 : Rendement annuel moyen ( $\mathrm{kg} / \mathrm{km} / \mathrm{an})$ estimé par secteur d'exploitation. Les diamètres des cercles sont proportionnels à la somme des rendements détaillés par catégorie de pêcheurs. En blanc, les professionnels ; en gris, les amateurs.

Figure 4 : Average annual yield $(\mathrm{kg} / \mathrm{km} / \mathrm{yr})$ estimated from the sector of exploitation. The diameter of the circles are proportional to the sum of the returns, classed by categories of fishermen. In white: the professionals ; in grey, the amateurs.

\section{Pêcheur aux carrelets}

Les pêcheurs aux carrelets fournissent 40 carnets utilisables pour le calcul des CPUE sur 44 rendus. Pour ce métier, tous les stocks du secteur cité au 2.4. répondent à nos conditions de définition des CPUE sauf le stock d'écrevisses américaines. Les In(CPUE) des pêcheurs aux carrelets ne sont pas significativement différentes de celles des autres métiers pour le stock de petits cyprinidés et de poissons-chats. En revanche, elles sont plus importantes pour ceux de grands cyprinidés, de sandres, de brochets, de perches et de silures ( $p \leq 5 \%$ au test $F$ de Scheffe). 
Tableau VI : ANOVA à un facteur du logarithme népérien des Captures par Unité d'Effort (CPUE en kg/jours mensuels) des pêcheurs professionnels testant l'effet de l'année pour les stocks les plus importants de la Saône aval. La moyenne annuelle et l'erreur standard (Err. St.) sont précisées. $\mathrm{N}$ : nombre de CPUE.

Table VI : One-way ANOVA of the log-transformed Catch Per Unit Effort (CPUE in $\mathrm{kg} /$ day for each month) of the commercial fishermen testing for differences among years for the most important stocks of the lower Saône River.The annual mean and the standard error (Err. St.) are given. $\mathrm{N}$ : number of CPUE.

\begin{tabular}{ccccc}
\hline & & & \multicolumn{2}{c}{ Moyenne de In(CPUE) \pm Err. St. } \\
\cline { 4 - 5 } STOCK & $N$ & Effet Année & 1988 & 1989 \\
\hline Poisson-chat & 69 & $\mathrm{p}=0,0452$ & $1,7 \pm 0,3$ & $1,0 \pm 0,2$ \\
Petits cyprinidés & 29 & $\mathrm{p}=0,1022$ & $1,5 \pm 0,4$ & $2,4 \pm 0,4$ \\
Ecrevisse americaine & 81 & $\mathrm{p}=0,6823$ & $1,2 \pm 0,3$ & $1,1 \pm 0,2$ \\
Grands cyprinidés & 82 & $\mathrm{p}=0,0270$ & $1,6 \pm 0,2$ & $1,0 \pm 0,2$ \\
Sandre & 60 & $\mathrm{p}=0,8511$ & $0,3 \pm 0,2$ & $0,3 \pm 0,2$ \\
Brochet & 64 & $\mathrm{p}=0,2628$ & $0,5 \pm 0,3$ & $0,1 \pm 0,2$ \\
Perche & 41 & $\mathrm{p}=0,5299$ & $-1,3 \pm 0,3$ & $-1,5 \pm 0,2$ \\
Silure & 30 & $\mathrm{p}=0,6379$ & $-0,6 \pm 0,3$ & $-0,4 \pm 0,4$ \\
\hline
\end{tabular}

Tableau VII : ANOVA à deux facteurs du logarithme népérien des Captures par Unité d'Effort (CPUE en kg/jours mensuels) des pêcheurs amateurs testant l'effet de l'année, du métier et de leur interaction pour les stocks les plus importants de la Saône aval. Les moyennes annuelles et par métier sont précisées avec leurs erreurs standards (Err. St.). N : nombre de CPUE.

Table VII : Two-way ANOVA of the log-transformed Catch Per Unit Effort (CPUE in $\mathrm{kg} / \mathrm{day}$ for each month) of the recreational fishermen testing for differences among years and categories of fishing and their interaction for the most important stocks of the lower Saône River.The mean by categories of fishing and the annual means are given with their standard errors (Err. St.). $\mathbf{N}$ : number of CPUE.

\begin{tabular}{|c|c|c|c|c|c|c|c|c|c|}
\hline \multirow[b]{2}{*}{ STOCK } & \multirow[b]{2}{*}{$N$} & \multirow[b]{2}{*}{ Effet Métier } & \multicolumn{3}{|c|}{ 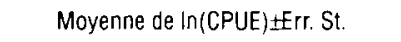 } & \multirow[b]{2}{*}{ Effet Année } & \multicolumn{3}{|c|}{ Moyenne de In(CPUE) \pm Err. St. } \\
\hline & & & Carrelet & $\begin{array}{l}\text { Carrelet } \\
+ \text { Piege }\end{array}$ & Piège & & 1988 & 1989 & Interaction \\
\hline $\begin{array}{l}\text { Poisson-chat } \\
\text { Petits cyprinidés } \\
\text { Ecrevisse americaine } \\
\text { Grands cyprinidés } \\
\text { Sandre } \\
\text { Brochet } \\
\text { Perche } \\
\text { Silure }\end{array}$ & $\begin{array}{l}655 \\
131 \\
618 \\
760 \\
396 \\
428 \\
463 \\
166\end{array}$ & $\begin{array}{l}p=0.0584 \\
p=0.0776 \\
p=0,0001 \\
p=0,0001 \\
p=0,0001 \\
p=0.0001 \\
p=0.0015 \\
p=0.0004\end{array}$ & $\begin{array}{r}0,1 \pm 0.1 \\
-0,02 \pm 0.1 \\
- \\
0,5 \pm 0,1 \\
0.02 \pm 0.1 \\
-0,1 \pm 0.1 \\
-0,6 \pm 0.1 \\
0.9 \pm 0,2\end{array}$ & $\begin{array}{r}0.3 \pm 0.1 \\
-0.2 \pm 0.1 \\
-0.5 \pm 0.1 \\
0.1 \pm 0.1 \\
-0.5 \pm 0.1 \\
-0.4 \pm 0.1 \\
-0.9 \pm 0.1 \\
-0.1 \pm 0.1\end{array}$ & $\begin{array}{c}0.3 \pm 0.1 \\
- \\
-0.04 \pm 0.1 \\
-0.6 \pm 0.1 \\
- \\
-0.7 \pm 0.1 \\
-1.0 \pm 0.1 \\
-0.4 \pm 0.2\end{array}$ & $\begin{array}{l}p=0,1015 \\
p=0.1754 \\
p=0,0003 \\
p=0,8409 \\
p=0.6311 \\
p=0.0011 \\
p=0,1568 \\
p=0.2108\end{array}$ & $\begin{array}{r}0,4 \pm 0,1 \\
-0,3 \pm 0,1 \\
-0,5 \pm 0,1 \\
0,2 \pm 0,1 \\
-0,2 \pm 0,1 \\
-0,4 \pm 0,1 \\
-0,8 \pm 0,1 \\
0,6 \pm 0,2\end{array}$ & $\begin{array}{r}0,2 \pm 0,1 \\
-0,1 \pm 0,1 \\
-0,2 \pm 0,1 \\
0,1 \pm 0.1 \\
-0,2 \pm 0,1 \\
-0,3 \pm 0.1 \\
-0,8 \pm 0.1 \\
-0,2 \pm 0.1\end{array}$ & $\begin{array}{l}p=0,2313 \\
p=0,2348 \\
p=0,0131 \\
p=0,6085 \\
p=0,0682 \\
p=0,0277 \\
p=0,0053 \\
p=0,5089\end{array}$ \\
\hline
\end{tabular}

\section{Pêcheur aux pièges}

Les pêcheurs aux pièges fournissent 17 carnets utilisables pour le calcul des CPUE sur 19 rendus. Les stocks de poissons-chats, d'écrevisses américaines, de grands cyprinidés et de silures répondent à nos conditions de définition des CPUE. Les In(CPUE) de ce métier sont généralement plus faibles que celles des autres métiers sauf pour les stocks de poissons-chats et d'écrevisses américaines. Pour ce dernier, les In(CPUE) sont plus importantes que celles des pêcheurs utilisant des carrelets et des pièges ( $p \leq 5 \%$ au test $t$ de Student). 


\section{Pêcheur aux carrelets et aux pièges}

Les pêcheurs utilisant des carrelets et des pièges présentent 34 carnets utilisables sur 37 rendus. Ils déclarent des prélèvements sur tous les stocks précités dans des conditions qui permettent le calcul des CPUE. Leurs In(CPUE) sont généralement intermédiaires entre celles des pêcheurs aux carrelets et des pêcheurs aux pièges. Les differrences avec les pêcheurs aux carrelets sont toutes significatives, au seuil alpha de $5 \%$, au test $F$ de Scheffe ou au test $t$ de Student sauf en ce qui concerne le stock de petits cyprinidés. Les différences avec les pêcheurs aux nasses sont également significatives, au seuil alpha de $5 \%$, sauf en ce qui concerne les stocks de poissons-chats, de silures et de perches.

\section{DISCUSSION ET CONCLUSION}

\subsection{Comparaisons avec les données antérieures}

Ces résultats évaluent pour la première fois simultanément les captures effectuées par les pêcheurs aux engins amateurs et professionnels sur le Rhône et la Saône. La production totale actuelle dépasse à peine les 235 tan de poissons consommées à Lyon, au début du siècle, en provenance du Rhône, de la Saône et du canal de Jonage selon SORNAY (1932). Elle représente $17 \%$ des 1530 t produites annuellement au milieu du siècle sur tout le Domaine Public Fluvial français selon BOISSET et VIBERT (1944). II n'existe pas, à notre connaissance, de statistiques récentes sur les captures des pêcheurs amateurs. La biomasse des captures professionnelles, estimée sur l'ensemble de l'espace étudié, s'inscrit dans la fourchette des valeurs obtenues par BABIN (1991) pour l'année 1988. Le détail, par déclaration brute, est semblable bien que les petits cyprinidés ainsi que les carnassiers et salmonidés soient moins abondants dans nos résultats (Fig. 3). En revanche, la biomasse moyenne estimée des captures d'anguilles par les professionnels sur le Rhône aval est très importante en comparaison avec les 11 tan d'anguilles indiquées anciennement dans un manuscrit anonyme cité par PATTÉE (1988) qui fait un bilan détaillé des productions du Rhône en aval de Lyon avant 1944. Malheureusement les précisions quant à la méthode employée sont particulièrement vagues sur le fac-similé présenté dans le Schéma de Vocation du fleuve Rhône (ANONYME, 1991a, p.16).

Contrairement à ce qui est observé dans d'autres bassins de grands cours d'eau européens (PETTS et al., 1989 ; DILL, 1990) ou français (BABIN, 1992a), les productions du bassin du Rhône ne sont pas dominées par le secteur estuarien et la pêche des migrateurs. Même s'il faut noter ici la forte imprécision sur les estimations des captures professionnelles du secteur concerné, l'absence de saumon en Méditerranée, la surpêche de l'esturgeon dès la fin du siècle dernier (GOURRET, 1897) et la diminution du stock d'aloses à partir de la fin des années quarante avec l'aménagement du Bas-Rhône (GALLOIS, 1947) explique certainement cette particularité. Le secteur fluvial de la Saône aval, qui n'a pas été récemment modifié par l'homme, présente les productions les plus importantes du bassin tant pour les amateurs que pour les professionnels. C'est une zone de première importance nationale pour la pratique de la pêche strictement fluviale.

Les rendements kilométriques obtenus, compris entre 44,5 et $558,1 \mathrm{~kg} / \mathrm{km} / \mathrm{an}$, sont nettement inférieurs aux estimations effectuées avant 1944 sur le Rhône, en aval de Lyon (PATTÉE, 1988), qui se situent entre 1105 et $1846 \mathrm{~kg} / \mathrm{km} /$ an suivant les secteurs lorsqu'on exclut l'alose, la lamproie et les autres migrateurs anadromes qui ne peuvent plus atteindre la majorité du bassin actuellement. Les captures par pêche à la ligne et le braconnage, qui comptent seulement pour $12 \%$ de ces statistiques anciennes, ne suffisent pas à expliquer la différence. En revanche, nos résultats se rapprochent des estimations récentes effectuées sur le Rhône qui situent le rendement entre 395 et $816 \mathrm{~kg} / \mathrm{km} / \mathrm{an}$ (BOUCHERON, 1980 ). Ils sont supérieurs aux $20 \mathrm{~kg} / \mathrm{km} / \mathrm{an}$ capturés sur le Danube autrichien (BACALBASA-DOBROVICI, 1989). Le rendement des professionnels est naturellement beaucoup plus élevé que celui des amateurs qui ne sont pas autorisés à utiliser la même puissance de pêche. Exploitée plus intensément que les autres secteurs, la Saône aval apparaît comme la partie la plus productive avec les meilleurs rendements du bassin. Sur le Rhône, les rendements surfaciques sont légèrement inférieurs aux 23 à $33 \mathrm{~kg} / \mathrm{ha} / \mathrm{an}$ estimés sur le cours supérieur du Mississippi (FREMLING et al., 1989). Ils sont très 
inférieurs aux $205 \mathrm{~kg} / \mathrm{ha} / \mathrm{an}$ de productivité théorique moyenne obtenus récemment sur 26 cours d'eau tempérés (WELCOMME, 1985). La pêche aux engins laisse donc globalement une large partie des stocks en place à la disposition d'autres modes d'exploitation.

II n'existe pas de données de références pour les CPUE, telles que nous les avons définies. La différence entre les CPUE des amateurs et des professionnels s'explique facilement à partir des distinctions de puissances de pêche déjà mises en évidence dans la première partie de l'article. Les différences internes, entre les métiers amateurs, peuvent être mises en relation avec les rendements pondéraux moyens journaliers des engins donnés dans la première partie de l'article. Les pièges ont globalement un rendement pondéral journalier plus faible que les carrelets. Les pêcheurs aux carrelets ont effectivement des CPUE plus élevées. Cependant, les pêcheurs utilisant exclusivement des pièges peuvent avoir des CPUE plus élevées pour des espèces comme l'écrevisse américaine ou le poisson-chat pour lesquelles les carrelets ne sont pas appropriés.

La plupart des variations inter-annuelles mises en évidence sont confirmées par Paul BUISSON, représentant des pêcheurs professionnels, en particulier dans le cas du poissonchat, qui semble avoir été victime d'un virus spécifique identifié comme responsable de mortalités massives dans la région durant cette période (POZET et al., 1992). En revanche, l'augmentation du stock d'ablettes, signalée par la même source, n'est pas sensible sur les In(CPUE) de petits cyprinidés.

\subsection{Précision et biais de la méthode}

Le nombre de réponses obtenues est suffisant pour estimer convenablement les biomasses prélevées par les pêcheurs dans l'hypothèse où les carnets reçus représentent un échantillon aléatoire simple. La vérification de cette hypothèse reste particulièrement difficile à effectuer (LAUREC et al., 1983). Néanmoins, la stratification de l'échantillonnage permet d'appréhender les structures de la pêcherie dans toute leur complexité. La précision est toujours meilleure pour les prélèvements amateurs car ces pêcheurs sont nombreux et leur taux de réponses suit une allocation proportionnelle à l'effectif des autorisations dans chaque strate d'échantillonnage (CHANGEUX et ZYLBERBLAT, ce numéro). Chez les professionnels, elle peut localement s'avérer très mauvaise en raison d'une forte variabilité inter-carnets (cas des professionnels du Rhône deltaïque, Tableau III). Ces caractéristiques statistiques ne nous préservent pas des déclarations erronées avec, par exemple, le signalement d'aloses dans la Saône amont par un amateur. Mais ce phénomène semble rester négligeable.

L'orientation de la pêche aux engins vers certaines espèces apporte un biais qui nous empêche de considérer ces statistiques comme une description fidèle du peuplement tant au niveau de la composition spécifique que de l'abondance des différentes espèces. De même, le regroupement de plusieurs espèces sous une même appellation limite la précision de cette description. Malgré ces limites, la répartition des captures entre secteurs ne conduit pas à la même carte factorielle que celle obtenue à partir des engins de pêche qui, dans la première partie de cet article, rassemblait le Rhône amont et la Saône amont (CHANGEUX et ZYLBERBLAT, ce numéro). Ainsi, les secteurs de la Saône amont et du Rhône amont fournissent-ils des captures différentes, malgré des modes d'exploitation semblables, alors que les secteurs du Rhône amont et du Rhône aval fournissent des captures semblables, malgré des modes d'exploitation différents. Les biais apportés par l'utilisation de différents engins n'empêchent pas de faire ressortir les différences écologiques fondamentales connues depuis longtemps entre la Saône et le Rhône (KREITMANN, 1932 ; LEGER et al., 1945).

La biomasse des captures mensuelles déclarées ne donne pas une image des variations des stocks en place, elle traduit avant tout les variations de l'effort de pêche. Par exemple, la pêche du muge se limite aux mois les plus froids car ces poissons deviennent inconsommables lorsqu'ils sont maintenus morts à des températures élevées. Les deux saisons de pêche "froides" et "chaudes", mises en évidence par l'analyse des engins, se singularisent par des compositions de captures particulières qui correspondent à la sélectivité des engins utilisés et aux périodes de fermeture (cas des salmonidés en hiver). En revanche, l'impact de la fermeture du mois de mai, nettement visible sur l'évolution de l'intensité de pêche estimée, ainsi que sur les variations de quantités capturées et la 
composition des captures, n'est pas décelable dans la composition de l'effort de pêche (CHANGEUX et ZYLBERBLAT, ce numéro). Les engins utilisés pendant ce mois ne se distinguent pas beaucoup de ceux utilisés pendant la période "intermédiaire". On observe ici les limites d'une approche globale de la pêcherie multi-spécifique et multi-engins lorsqu'on ne fait pas de distinction entre les différents secteurs et les différents métiers. II font donc séparer les captures suivant les secteurs et les métiers pour standardiser l'effort de pêche.

L'utilisation des combinaisons d'engins proposée dans la première partie de l'article pour définir les différents métiers de pêche s'avère justifiée puisque les CPUE varient généralement de manière significative entre eux. Ces distinctions correspondent bien à des hétérogénéités dans la pêcherie qu'il est nécessaire de prendre en compte pour standardiser les CPUE. En revanche, l'emploi des CPUE comme indice d'abondance annuel des stocks fait de nombreuses hypothèses que nos données ne permettent pas de vérifier. Une première hypothèse concerne le nombre de jours de pêche qui est assimilé au nombre de jours avec captures. Cela est certainement peu éloigné de la vérité vu l'efficacité des engins utilisés. Une seconde hypothèse suppose que, pour chaque métier, une espèce donnée est recherchée avec la même intensité d'une année sur l'autre. La concordance des évolutions obtenues indépendamment sur une même capture avec les CPUE de plusieurs métiers différents offre une possibilité d'éprouver la signification de ces CPUE en tant qu'indice d'abondance annuel. II faut néanmoins veiller à ce que ces métiers soient comparables. Par exemple, la différence entre les efforts de pêche des professionnels et des amateurs rend leurs CPUE difficilement comparables, si on ne prend pas en compte le nombre d'engins. De plus, certains engins n'ont pas les mêmes caractéristiques (e.g. les filets des pêcheurs amateurs sont plus petits, les professionnels sont les seuls à utiliser des pièges de grandes tailles et petites mailles selon CHANGEUX et ZYLBERBLAT (ce numéro)). II n'y a d'ailleurs pas de variation significative commune des CPUE entre les deux catégories de pêcheurs. En revanche, les différents métiers amateurs montrent pour l'écrevisse américaine et le brochet des variations communes significatives mais ces variations s'accompagnent d'effets d'interaction importants. Ce qui veut dire que les In(CPUE) ne varient pas d'une année sur l'autre avec la même importance suivant les métiers. Cet effet d'interaction agit également de manière significative sur les CPUE de perches et dans une moindre mesure sur celles de sandres. La représentation des moyennes des In(CPUE) par année et par métier permet de visualiser ces effets (Fig. 5). Les In(CPUE) des amateurs utilisant des pièges et des carrelets varient peu d'une année sur l'autre contrairement à celles des pêcheurs utilisant un seul type d'engins. L'utilisation d'une combinaison d'engins amortit donc les variations observées. Un métier plus spécialisé présente des variations plus importantes. L'effet d'interaction, peut également intervenir pour dissimuler des évolutions importantes d'une année sur l'autre dans un métier particulier. Ainsi, la moyenne des In(CPUE) de perche diminue-t-elle chez les amateurs aux carrelets tandis qu'elle augmente chez les amateurs aux pièges. II y a ici probablement une différence entre les stocks exploités à préciser par une étude des caractéristiques biologiques des individus capturés pour chaque type d'engins.

\subsection{Apports de l'étude des captures par pêche aux engins}

Les statistiques de pêche aux engins constituent un procédé d'échantillonnage particulièrement performant en complément des pêches scientifiques pour évaluer les ressources piscicoles dans les grands cours d'eau.

La pêche électrique, telle qu'elle est couramment utilisée pour l'échantillonnage scientifique des poissons des petits cours d'eau, porte l'essentiel de son effort sur les juvéniles lorsqu'elle est transposée dans les grands cours d'eau (PONT et al., 1992). Or il s'agit de la fraction la plus fluctuante du peuplement. Ainsi, les données recueillies de cette manière fournissent-elles une bonne évaluation du recrutement, mais ne donnent pas une bonne image du peuplement en place. Pour les grands cours d'eau, l'utilisation d'engins (senne, filets multi-mailles, nasses...) reste encore le meilleur procédé d'échantillonnage mais ces techniques sont particulièrement difficiles à mettre en œuvre. Les carnets de pêche rendent compte de ce type de pratiques, mais il était nécessaire d'évaluer la cohérence des données fournies par ce procédé avant d'en faire un emploi systématique. 


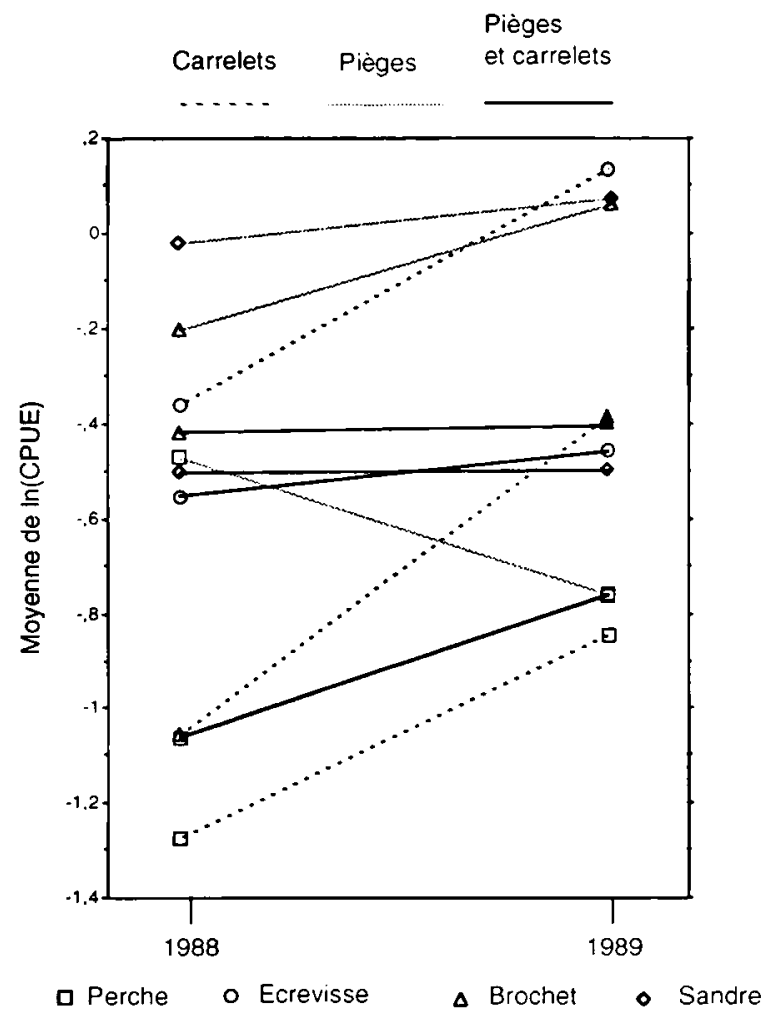

Figure 5 : Moyenne annuelle du logarithme des Captures Par Unité d'Effort (CPUE en kg/jours mensuels) des pêcheurs amateurs pour les stocks montrant une interaction significative entre l'année et le métier. Les In(CPUE) des pêcheurs utilisant des pièges et des carrelets simultanément sont moins variables.

Figure 5 : Average annual log-transformed Catch Per Unit Effort (CPUE in $\mathrm{kg} / \mathrm{day}$ for each month) for recreational fishermen, using only the stocks showing a significant interaction between year and category of fishing. The In(CPUE) of fishermen using both traps and lift-nets are less variable.

Contrairement aux tentatives précédentes qui étaient limitées dans le temps (GUILLOUET, 1988 ; ANONYME, 1989), notre étude montre que des résultats significatifs sur l'estimation des prélèvements et l'utilisation des CPUE peuvent être obtenus dès la deuxième année, en particulier grâce aux carnets des pêcheurs amateurs. Même si les pêcheurs recherchent particulièrement certaines espèces cibles, les distinctions écologiques entre les différents secteurs persistent. Lorsque ces espèces sont également relativement rares dans le peuplement (e.g. la lamproie marine, l'alose feinte, le silure glane ou les salmonidés) les carnets de pêche, en mettant à la disposition des scientifiques et des gestionnaires des biomasses importantes, sont actuellement les seuls à pouvoir fournir des informations significatives. Par exemple, la localisation des catégories de captures professionnelles met en évidence la présence de poissons amphihalins dans le Rhône aval (Tableau III), en amont du barrage de Beaucaire. Ce barrage était jusqu'ici considéré comme un obstacle difficilement franchissable pour les migrateurs, en particulier pour les aloses (LARINIER et al., 1978 ; RAMEYE et al., 1976). Ces captures sont à l'origine d'études récentes qui ont confirmé la possibilité d'un passage par l'écluse de navigation (ANONYME, 1991b). Cependant, le barrage reste un obstacle majeur car la quantité d'aloses déclarée en amont est négligeable face aux estimations anciennes $(50,5 \mathrm{~kg}$ en deux ans contre 34 tan avant 1944 selon PATTÉE, 1988). De même, les carnets de pêche révèlent la présence non négligeable des salmonidés dans le Rhône amont, et une répartition préférentielle du silure dans la Saône et dans le Rhône deltaïque. 


\subsection{Perspectives}

Dans la perspective d'un suivi à long terme, il sera possible d'améliorer la précision de nos estimations en tirant parti de la forte corrélation inter-annuelle des données. Les variations inter-annuelles pourront également être affinées, d'une part, en effectuant des comparaisons mois par mois de manière à tirer parti du rythme saisonnier de certaines pêches, d'autre part, en utilisant des méthodes statistiques plus adaptées au traitement des variables non normales (modèle log normal et tests par permutations) comme le proposent CRYER et MACLEAN (1991). De ce point de vue, ce sont certainement les données fournies par les nombreux pêcheurs amateurs qui restent le plus facilement exploitables. Mais il est possible d'améliorer nos connaissances en effectuant quelques modifications.

D'abord, sous leur forme actuelle, les données limitent le calcul des CPUE à l'échelle mensuelle, en kg/jour de pêche. Pour certaines espèces et certains métiers, en particulier ceux de pêche professionnelle, il serait intéressant de pouvoir disposer, au moins sur un échantillon représentatif par strate, des captures au jour le jour de manière à prendre en compte les variations journalières des CPUE. Pour augmenter la fiabilité des CPUE, la connaissance, au moins mois par mois, des espèces recherchées est également nécessaire. II serait également souhaitable de disposer du nombre d'engins utilisés, avec plus de précision, de manière à pouvoir exploiter dans le détail les CPUE fournies par les métiers multi-engins.

Ensuite, ces statistiques pourraient être analysées en faisant intervenir les principes de la dynamique des populations si elles étaient complétées par des informations concernant l'âge, la taille et le sexe des captures les plus recherchées.

Enfin, il serait également souhaitable d'intégrer les captures effectuées sur le Doubs exploité par 7 pêcheurs professionnels selon CASTELNAUD et BABIN (1992). Ce secteur, ignoré de nos statistiques, semble être important à l'échelle du bassin (ANONYME, 1991C).

En conclusion, cette méthode apparaît comme un moyen particulièrement efficace pour suivre l'évolution du peuplement piscicole des grands cours d'eau dans le cadre d'une surveillance biologique des milieux aquatiques. Dans l'avenir, elle pourrait permettre d'évaluer la mortalité par pêche dans un modèle de dynamique des populations qui devrait nécessairement prendre en compte les autres impacts anthropiques. En complément des connaissances sur les autres hydrosystèmes français déjà inventoriés, elle peut d'ores et déjà servir de base pour orienter la recherche en écologie fluviale. Son extension aux pêcheurs à la ligne est possible, avec un minimum d'aménagements comme aux Etats-Unis ou au Royaume-Uni (MALVESTUDO, 1983 ; HICKLEY et STARKIE, 1985 ; COWX et al., 1986).

\section{REMERCIEMENTS}

Nous tenons à remercier ici tous les pêcheurs aux engins et leurs associations, ainsi que le Service de la Navigation Rhône-Saône, la Direction Régionale de l'Environnement Rhône-Alpes et les différents services gestionnaires sans lesquels la base de ce travail n'aurait pu être établie. Nous remercions également Paul BUISSON (Président de la Coordination Nationale de la Pêche Professionnelle en Eaux Douces) pour ses informations complémentaires concernant l'état des stocks, Philippe HENSEL pour ses traductions en anglais, ainsi que Didier PONT et Pierre ELIE pour leur aide dans la rédaction. Ce travail fait partie du programme interdisciplinaire de recherches sur l'environnement du CNRS. II a été financé par une bourse de Docteur Ingénieur du CNRS.

\section{BIBLIOGRAPHIE}

ANONYME, 1989. La pêche professionnelle et amateur aux engins. Schéma de vocation piscicole du fleuve Rhône, Document n 7, DBRMC, juillet 1989, $41 \mathrm{p}+$ annexes.

ANONYME, 1991a. Rapport de synthèse. Schéma de vocation piscicole du fleuve Rhône, DBRMC/SNRS/Min. de l'environnement, juillet 1991, $202 p+$ annexes.

ANONYME, 1991b. Suivi de la migration des aloses. Schéma de vocation piscicole du fleuve Rhône, Document n 4, DBRMC/SNRS/Min. de l'environnement, février 1991, $7 p+$ annexes. 
ANONYME, 1991c. Eaux de Rhône Méditerranée Corse. Agence de l'eau RMC, Lyon, 331 p.

ANONYME, 1993. Etude du peuplement piscicole et de la pêche amateur et professionnelle aux engins. Schéma de vocation piscicole de la Saône, Groupement des Fédérations de pêche de l'Ain, de la Côte-d'Or, du Rhône, et de la Saône-et-Loire, février 1993, $49 \mathrm{p}+$ annexes.

BABIN D., 1991. Résultats de l'enquête express 1989 - Estimation de la production et du chiffre d'affaires des pêches professionnelles continentales françaises (Marins pêcheurs exclus). CEMAGREF/Division ALA, Bordeaux (France), février 1991, $4 \mathrm{p}$.

BABIN D., 1992a. Contribution à l'étude d'une ressource naturelle renouvelable : la pêche professionnelle en eau douce en France. Thèse de doctorat, Univ. Rennes 2, 212 p. + annexes.

BABIN D., 1992b. Atlas de la pêche professionnelle en eau douce en France. Annexe de la Thèse de doctorat, Univ. Rennes 2, pages non numérotés.

BACALBASA-DOBROVICI N., 1989. The Danube river and its fisheries in DODGE D.P. Proceedings of The International Large River Symposium, 455-468, Can. Spec. Publ. Fish. Aquat. Sci. $n^{\circ} 106$.

BOISSET L.de et VIBERT R., 1944. La pêche fluviale en France, son état, son avenir. Librairie des Champs-Elysées, Paris, $277 \mathrm{p}$.

BOUCHERON G., 1980. Estimation de dommage piscicole consécutif à la pollution du Rhône par les vidanges des barrages de Verbois et Genissiat, en juin 1978. Rapport CTGREF, Thonon-les-bains (France), $18 \mathrm{p}$.

BRAVARD J.P., 1987. Le Rhône, du Léman à Lyon. La Manufacture, Lyon, 452 p.

CASTELNAUD G. et BABIN D., 1990. La pêche professionnelle aux filets et aux engins dans les eaux continentales françaises, deuxième partie : les bassins Rhône-Saône-Doubs, Rhin, Somme, Charente, Garonne-Dordogne, Adour et les lacs alpins. Rapport du CEMAGREF/Division ALA, décembre 1990, Bordeaux (France), 141 p + annexes.

CASTELNAUD G. et BABIN D., 1992. La pêche professionnelle fluviale et lacustre en France. Enquête au fil de l'eau. Etudes ressources en eau $n^{\circ} 5$, Min. de l'Environnement, CEMAGREF/Division ALA, Bordeaux (France), 291 p.

CHANGEUX T. et ZYLBERBLAT M., ce numéro. Analyse des statistiques de pêche aux engins dans le bassin du Rhône. Première partie : étude de l'effort de pêche. Bull. Fr. Pêche Piscic., 330.

CHESSEL D. et DOLEDEC S., 1991. ADE Software, version 3.1., URA CNRS 1451, Université $\mathrm{Cl}$. Bernard, Lyon (France).

COWX I.G., FISHER K.A.M. et BROUGHTON N.M., 1986. The use of angler's catches to monitor fish populations in large water bodies, with particular reference to the River Derwent, Derbyshire, England. Aquaculture and Fisheries Management, 17, 95-103.

CRYER M. et MACLEAN G.D., 1991. Catch for effort in a New Zealand recreational trout fishery - a model and implication for survey design in COWX I.G. Catch effort sampling strategies, 61-71, Fishing news books, Oxford (U.K.).

DILL W.A., 1990. Inland fisheries of Europe. EIFAC Technical Paper n52, FAO Publ., Rome, $471 \mathrm{p}$.

FREMLING C.R., RASMUSSEN J.L., SPARKS R.E., COBB S.P., BRYAN C.F. et CALFLIN T.O., 1989. Mississippi river fisheries : a case history in DODGE D.P. Proceedings of The International Large River Symposium, 309-351, Can. Spec. Publ. Fish. Aquat. Sci. $\mathrm{n}^{\circ} 106$.

GALLOIS C., 1947. Aménagement du Rhône Vivarais par la Compagnie Nationale du Rhône. Ses incidences sur l'économie piscicole. Mesures tendant à les atténuer. Bull. Fr. Piscic., 146, 25-34.

GOURRET P., 1897. Les étangs saumâtres du Midi de la France et leurs pêcheries. Annales du Musée d'Histoire naturelle de Marseille, 5(1), 127-200. 
GUILLOUET J., 1988. Possibilité d'utilisation des données halieutiques pour l'estimation de paramètres des populations piscicoles. Rapport technique de DEA Université $\mathrm{Cl}$. Bernard, Lyon (France), $20 \mathrm{p}$.

HICKLEY P. et STARKIE A., 1985. Cost effective sampling of fish populations in large water bodies. J. Fish Biol., 27(A), 151-161.

KREITMANN L., 1932. Les grandes lignes de l'économie piscicole du bassin français du Rhône. Trav. Lab. Hydrobiol. Pisc. Grenoble, 24, 127-131.

LARINIER M., RIVIER B., ALLARDI J. et TROCHERIE F., 1978. Possibilité de franchissement du seuil de Beaucaire par les aloses du Rhône. Bull. Fr. Piscic., 268, 107-120.

LAUREC A. et LE GUEN J.C., 1981. Dynamique des populations marines exploitées, tome I : concepts et modèles. Rapports scientifiques et techniques $n^{\circ} 45$, C.N.E.X.O, Brest, novembre 1982 ( $2^{\mathrm{e}}$ édition), $118 \mathrm{p}$.

LAUREC A., LE GUEN J.C. et FRONTIER S., 1983. Collecte de l'information pour une gestion rationnelle des stock halieutiques in FRONTIER S., Stratégie d'échantillonnage en écologie, 385-415, Masson, Paris.

LEGER L., BURDIN M.G. et ARNAUD M.A., 1945. Etude sur l'hydrobiologie et l'économie piscicole du département du Rhône. Travaux du laboratoire d'Hydrobiologie et de Pisciculture de l'Université de Grenoble (France), $15 p+1$ carte.

MALVESTUDO S.P., 1983. Sampling the recreational fisheries in NIELSEN L.A. \& JOHNSON D.L., Fisheries Techniques, 397-419, American Fisheries Society Publication, Bethesda (USA).

PATTEE E., 1988. Fish and their environment in large european river ecosystems : The Rhône. Sciences de l'Eau, 7(1), 35-74.

PETTS G.E., MOLLER H. et ROUX A.L., 1989. Historical changes of large alluvial rivers: western europe. John Wiley \& Son Ltd, Chichester, $355 \mathrm{p}$.

PONT D., TORRE F., POIZAT G., 1992. Etablissement d'une méthodologie pour un suivi piscicole du Rhône, rapport à la Délégation de Bassin RMC et à Agence de l'eau RMC, Janvier 1992, $55 \mathrm{p}+$ annexes.

POZET F., MORAND M., MOUSSA A., TORHY C. et KINKELIN P. de, 1992. Isolation and preliminary characterization of a pathogenic icosahedral deoxyribovirus from the catfish Ictalurus melas. Dis. aquat. Org., 14, 35-42.

RAMEYE L., KIENER A., SPILLMANN C.P. et BIOUSSE J., 1976. Aspects de la biologie de l'alose du Rhône. Pêche et difficultés croissantes de ses migrations. Bull. Fr. Piscic., 263, 50-76.

ROUX M., 1985. Algorithmes de classification. Masson, Paris, $150 \mathrm{p}$.

SAPORTA G., 1990. Probabilités, analyse des données et statistiques. Tecnip, Paris, $493 \mathrm{p}$.

SCHERRER B., 1983. Techniques de sondage en écologie in FRONTIER S., Stratégie d'échantillonnage en écologie, 65-162, Masson, Paris.

SORNAY, 1932. La consommation du poisson d'eau douce à Lyon. Bull. Fr. Piscic, 54, 180-185.

THIOULOUSE J., 1991. MacDendro@, logiciel de classification hiérarchique, version 3.02, URA CNRS 243, Université Cl. Bernard, Lyon (France).

WELCOMME R.L., 1985. River fisheries. FAO Fish. Tech. Paper, 262, 330 p. 